\title{
Effects of Confinement on Critical Adsorption: Absence of Critical Depletion for Fluids in Slit Pores.
}

\author{
A. Maciołek ${ }^{1}$ and R.Evans \\ H.H. Wills Physics Laboratory, University of Bristol, \\ Bristol BS8 1TL, UK \\ N.B. Wilding \\ Department of Physics and Astronomy, University of Edinburgh \\ Edinburgh EH9 3JZ, U.K.
}

\begin{abstract}
The adsorption of a near critical fluid confined in a slit pore is investigated by means of density functional theory and by Monte Carlo simulation for a Lennard-Jones fluid. Our work was stimulated by recent experiments for $\mathrm{SF}_{6}$ adsorbed in a mesoporous glass which showed the striking phenomenon of critical depletion, i.e. the adsorption excess $\Gamma$ first increases but then decreases very rapidly to negative values as the bulk critical temperature $T_{c}$ is approached from above along near-critical isochores. By contrast our density functional and simulation results, for a range of strongly attractive wall-fluid potentials, show $\Gamma$ monotonically increasing and eventually saturating as the temperature is lowered towards $T_{c}$ along both the critical $\left(\rho=\rho_{c}\right)$ and sub-critical isochores $\left(\rho<\rho_{c}\right)$. Such behaviour results from the increasingly slow decay of the density profile away from the walls, into the middle of the slit, as $T \rightarrow T_{c}^{+}$. For $\rho<\rho_{c}$ we find that in the fluid the effective bulk field, which is negative and which favours desorption, is insufficient to dominate the effects of the surface fields which favour adsorption. We compare this situation with earlier results for the lattice gas model with a constant (negative) bulk field where critical depletion was found. Qualitatively different behaviour of the density profiles and adsorption is found in simulations for intermediate and weakly attractive wall-fluid potentials but in no case do we observe the critical depletion found in experiments. We conclude that the latter cannot be accounted for by a single pore model.

PACS numbers: 64.60.Fr, 05.70.Jk, 68.35.Rh, 68.15.+e
\end{abstract}

\section{INTRODUCTION}

The term 'critical depletion' was introduced in connection with experiments designed to study the phenomenon of critical adsorption for a pure fluid at a solid substrate [1,2]. When a fluid is brought to its bulk critical point in the presence of an attracting external wall or substrate, for example along a critical isochore, the amount adsorbed (adsorption) diverges as $\tau \equiv\left(T-T_{c}\right) / T_{c} \rightarrow 0 . T_{c}$ is the critical temperature. Theory [3] attributes these divergences to the fact that the wall causes a perturbation of the order parameter $(\mathrm{OP})$ profile $m(z) \equiv \rho(z)-\rho_{c}$, where $\rho(z)$ is the density profile, to extend over a distance $\sim \xi$, the bulk correlation length, from the surface. Close to criticality, where $\xi \sim|\tau|^{-\nu}$ ( $\nu$ is the critical exponent), the OP profile differs from its bulk value (fixed by the properties of the reservoir far from the substrate) over macroscopic distances and the adsorption can be a diverging quantity. Fisher and de Gennes [3] postulated that near criticality the OP profile should be described in terms of a universal scaling function, i.e. sufficiently close to $T_{c}$ and for sufficiently strongly attracting walls

$$
m(z)=\tau^{\beta} \mathcal{N}\left(\frac{z}{\xi}\right)
$$

where $\mathcal{N}$ is a universal scaling function and argued that as $\tau \rightarrow 0$ along the critical isochore $\left(\rho=\rho_{c}\right)$ the adsorption $\Gamma$ takes on the asymptotic, universal form

$$
\Gamma \equiv \int_{0}^{\infty} m(z) d z \sim \tau^{\beta} \xi \sim \tau^{\beta-\nu}
$$

where $z$ is the distance measured normal to the substrate, located at $z=0 . \beta$ is the critical exponent describing the vanishing of the bulk OP and $\beta-\nu \approx-0.305$ for Ising magnets or fluids. In Ref. [1] measurements were performed for $\mathrm{SF}_{6}$ adsorbed on a finely divided (colloidal) graphite adsorbent (Vulcan $3 \mathcal{G}$ ). The volumetric method employed in these experiments measured $\Gamma$ directly on approaching the critical point along the critical or near-critical isochores. Although $\Gamma$ increased as $\tau$ was reduced, consistent with (1.2), close to $T_{c}\left(\tau \sim 5 \times 10^{-3}\right)$ the adsorption reached a maximum and decreased sharply taking on negative values very close to $T_{c}$. Microgravity experiments by the same group confirmed these results [1,2. The critical depletion of $\Gamma$ was attributed to the confining effects of colloidal 
particles on the near critical fluid and new experiments, designed to test this conjecture, were performed on the sorption of $\mathrm{SF}_{6}$ in a mesoporous glass CPG-350, which comprises a rigid interconnected system of mesopores with a nominal pore diameter of $31 \mathrm{~nm}$. For the rigid porous material the measured adsorption showed a very similar temperature dependence to that found for the colloidal graphite adsorbent.

These experimental results have stimulated several simulation [4, 5 ] and theoretical studies [6,7] aimed at understanding the origin of the striking behaviour of $\Gamma$ and, in particular, to answer the fundamental question as to whether critical depletion is a single pore phenomenon, as is for example capillary-condensation [8] which arises from the combined effects of wall-fluid forces and finite-size and which manifests itself in simple confining geometries. The grand canonical Monte Carlo simulations [4.5 of a Lennard-Jones fluid confined between two structureless, attractive planar walls did indicate that the average, local density in the middle of this slit-pore could fall below $\rho_{c}$ under near-critical conditions thereby leading to depletion. However, it has been shown recently that the depletion found in [4, 5 ] is an artifact of the simulation procedure [27]. The theoretical studies [6] of the simplest model of a confined fluid, namely the lattice gas subject to identical surface fields located at the two walls, revealed a physical mechanism which could cause a dramatic decrease of the adsorption $\Gamma$ on approaching $T_{c}$ along near-critical isochores. Consider the situation when the density of the bulk reservoir is slightly lower than critical. Transcription of the lattice gas into the Ising model sets the chemical potential difference

$$
\Delta \mu \equiv \mu(\rho, T)-\mu\left(\rho_{c}, T\right)=2 H,
$$

where $\mu\left(\rho_{c}, T\right)$ is the chemical potential on the critical isochore and $H$ is the bulk magnetic field. Thus for $\rho<\rho_{c}$, $\Delta \mu=2 H<0$ and the bulk field favours the dilute (gas) phase. If the surface fields are sufficiently attractive that they favour adsorption of the dense (liquid) phase then one has a competition between bulk and surface fields which influences the shape of the OP profile and hence the behaviour of the adsorption in the slit-pore. When the pore is large and when the bulk correlation length is much smaller than the width of the pore $L$, then the fluid in the middle part of the pore should behave as a bulk fluid. For weak $H$, the bulk OP (magnetization) in the critical region behaves as

$$
m_{b}=H \chi \sim H \tau^{-\gamma}
$$

where $\chi$ is the susceptibility. Near the walls, on the other hand, the fluid should behave as in the semi-infinite near critical system subject to the surface field. 'Bulk' and 'surface' fields give diverging contributions, but of opposite sign, to the adsorption $\Gamma$ for $\tau \rightarrow 0$. For large pore widths and for $\tau \gg \tau_{0}$, where $\tau_{0}$ is defined by $\xi\left(\tau_{0}\right) \approx L$, the adsorption can be approximated, for $H<0$, by

$$
\Gamma \sim \mathcal{A}_{1} \tau^{\beta-\nu}-\mathcal{A}_{2}|H| \tau^{-\gamma} L,
$$

where $\mathcal{A}_{1}$ and $\mathcal{A}_{2}$ are positive amplitudes. Since $\gamma>(\nu-\beta)$ the second term always dominates for $T$ sufficiently close to $T_{c}$ provided $|H|$ is sufficiently strong and, as a consequence, depletion of $\Gamma$ will occur. For even smaller $\tau$, such that $\tau \ll \tau_{0}$, the adsorption saturates at a value which depends on $H$ and on the surface field. The lattice gas model of a single pore predicts critical depletion for $\rho<\rho_{c}$ only. For the case of the critical isochore $\rho=\rho_{c}$, the bulk field $H=0$ and as $\tau$ is reduced $\Gamma$ first increases monotonically following the Fisher-de Gennes power law and eventually saturates taking on a positive value at $\tau=0\left(T=T_{c}\right)[6]$.

This scenario was confirmed by explicit mean-field lattice gas calculations [6] and for two dimensional Ising films, with bulk and surface fields of opposite sign, by density matrix renormalization group calculations [7]. In the latter case it was shown that the near-critical fluctuations can lead to an even richer variation of $\Gamma(\tau)$. For certain values of $H$, in addition to the maximum, a minimum of $\Gamma$ appears where the correlation length approaches the pore width and the competition between the effect of adsorbing walls and the large susceptibility of the central region (favouring the dilute phase) becomes particularly strong. For weak $H<0$ the results for $\Gamma(\tau)$ obtained in the lattice gas (Ising) model of a single pore look very similar to those measured in the experiments of Ref. [1] and [2]. In the experiments for $\mathrm{SF}_{6}$ adsorbed in the controlled pore glass the actual isochores correspond to densities lower than critical, i.e. $\rho / \rho_{c}=0.995$ and 0.999 , so that $\Delta \mu<0$.

It is tempting then to argue that since $\Delta \mu<0$ there is an effective bulk field $H<0$ which competes with the surface fields to give rise to critical depletion. Although this is a plausible explanation of the observed phenomenon it does not take into account the actual situation in a fluid. For example, if the bulk density $\rho$ is fixed according to the experimental condition of the fluid reservoir, $\Delta \mu$ as defined by (1.3) will vary as $T$ approaches $T_{c}$. The corresponding bulk magnetic field will vary in the same way. In the present paper we show that taking into account the temperature dependence of $\Delta \mu$ has a profound effect on the behaviour of confined fluids near $T_{c}$. In particular we find that under the experimental conditions of Ref. [1,2] a simple fluid confined in a single slit pore should not exhibit critical depletion. Rather the adsorption should increase monotonically as $\tau \rightarrow 0$. This implies that an explanation of the experimental observations is still lacking. 
Our paper is organized as follows. In Sec.2 we reconsider the physical mechanism which leads to critical depletion of adsorption in the case of the lattice gas model of a pore considered in Ref. [6] and give a heuristic argument as to why this phenomenon should not be expected for real fluids when the reservoir density is fixed according to experimental conditions. Our argument is supported by explicit calculations of $\Gamma$ using the density functional approach and by grand canonical Monte Carlo simulations of the Lennard-Jones fluid in a slit-pore. In Sec. III] we report density functional results obtained from a square gradient approximation to the free energy functional and short-ranged (contact) wall-fluid potentials. Both classical and non-classical bulk free-energy densities are employed and and in the classical case we investigate two forms of the free energy density, namely the Landau model free energy and the free energy of the Lennard-Jones fluid as obtained in Ref. [9] from an accurate fit to simulation data. Sec. 4 describes the computer simulations of the density profiles and adsorption of the Lennard-Jones fluid on the critical isochore and for a sub-critical isochore $\rho<\rho_{c}$. Results are presented for various strengths of the 4-10 and 3-9 wall-fluid potentials. We conclude in Sec.5 with a discussion of the relevance of our findings for the experiments described earlier.

\section{HEURISTIC ARGUMENT.}

Here we reconsider the scaling argument [6] that predicts critical depletion in the lattice gas model of a single pore and modify it to incoroprate two features that are relevant for the case of fluids.

First, in order to mimic the experimental situation more closely one should account for the fact that $\Delta \mu \equiv$ $\mu(\rho, T)-\mu\left(\rho_{c}, T\right)$ will vary as the temperature $T$ approaches $T_{c}$ at constant $\rho$. This implies that the corresponding bulk magnetic field $H$ should also vary with $T$ and therefore that the second term of the approximate formula (1.5) for the adsorption $\Gamma$ might have a different $\tau$ dependence. This in turn may affect the result of the competition between the bulk and surface fields.

Second the lattice gas model considered in Ref. [6] has an exact particle-hole symmetry, which corresponds to the trivial symmetry under reversal of the field $H$ in the equivalent Ising model. For real fluids such symmetry is only approximate. It is well established that the reduced symmetry of fluids leads to scaling field mixing close to the critical point [10]. To linear order in $\tau$ and in $\mu(\rho, T)-\mu_{c}$, where $\mu_{c} \equiv \mu\left(\rho_{c}, T_{c}\right)$, the scaling fields have the form

$$
\begin{gathered}
u_{H} \equiv \mu(\rho, T)-\mu_{c}-c_{1} \tau \\
u_{\tau} \equiv \tau+c_{2}\left(\mu(\rho, T)-\mu_{c}\right),
\end{gathered}
$$

where the parameters $c_{1}$ and $c_{2}$ are system-dependent (non-universal) quantities controlling the degree of field mixing. $c_{1}$ is identified as the limiting critical slope of the coexistence curve, i.e. $c_{1} / T_{c}=\lim _{T \rightarrow T_{c}} d \mu^{\text {coex }}(T) / d T$. In order to account for the asymmetry of a real fluid near its critical point one should identify the bulk field $2 H$ with $u_{H}$ rather than with $\Delta \mu$ in the scaling analysis.

The temperature dependence of the bulk field $H$ depends on the particular equation of state. Consider first the the simplest possibility, i.e. the classical equation of state in the critical region given by retaining only the leading terms of the expanded van der Waals (vdW) equation of state. In terms of reduced temperature $\tau$ and density $r \equiv\left(\rho-\rho_{c}\right) / \rho_{c}$ the vdW equation of state reads

$$
\Delta \mu^{*}=-6 r-\frac{8}{3}(1+\tau) \ln \frac{1-r / 2}{1+r}+4(1+\tau)\left[\frac{1}{1-r / 2}-1\right]
$$

where $\Delta \mu^{*}=\Delta \mu / P_{c} v_{c}, P_{c}$ is the critical pressure and $v_{c}$ is the critical volume per molecule. The leading order behaviour of this equation in the near critical region is

$$
\Delta \mu^{*}=6 r \tau+\frac{3}{2} r^{3}
$$

where we have ignored terms $O\left(r^{4}\right)$ and $O\left(\tau r^{3}\right)$ and higher. Note that Eq. 2.4 exhibits particle - hole symmetry in that $\Delta \mu^{*}$ along an isotherm is antisymmetric with respect to the critical isochore

$$
\Delta \mu^{*}(-r, \tau)=-\Delta \mu^{*}(r, \tau)
$$

Moreover, for this case there is no scaling field mixing and $u_{H}=\Delta \mu^{*}$ whose magnitude decreases linearly in $\tau$ as $T_{c}$ is approached at constant $r$.

In order to analyse the influence of a $\tau$-dependent bulk field on the behaviour of the adsorption $\Gamma(\tau)$ we reconsider the approximate formula (1.5). For the expanded vdW equation of state (2.4) and $r<0\left(\rho<\rho_{c}\right)$ Eq. 1.5) becomes 


$$
\Gamma \sim \mathcal{A}_{1} \tau^{\beta-\nu}-\mathcal{A}_{3}|r|^{3} \tau^{-\gamma} L-\mathcal{A}_{4}|r| L,
$$

where $\mathcal{A}_{3}=(3 / 4) \mathcal{A}_{2}, \mathcal{A}_{4}=3 \mathcal{A}_{2}$ and for consistency with the vdW approach the critical exponents should take on their classical values, $\beta=\nu=1 / 2, \gamma=1$ and the first (critical adsorption) term diverges as - $\ln \tau$. Apart from the additional temperature independent term, Eq.(2.6) has the same form as for a constant bulk field (Eq.(1.5)). The additional term does not affect the shape of the curve $\Gamma(\tau)$. It simply shifts $\Gamma(\tau)$ as a whole towards negative values and for large widths of the pore $L$ and (or) relatively large $|r|$ it could drive $\Gamma$ negative sufficiently far from the critical point (large $\tau$ ). Closer to $T_{c}$ the temperature dependent terms dominate and the analysis of $\Gamma(\tau)$ as a function of $H$ and $L$ performed in Ref. [6] for constant bulk field $H$, goes through with $|H|$ replaced by $|r|^{3}$. Following Ref. [6] we rewrite Eq.(2.6) in the form

$$
\Gamma(\tau)=\tau^{\beta-\nu}\left[\mathcal{A}_{1}-\left(\frac{\tau}{\tau_{r}}\right)^{\nu-\Delta}\right]-\mathcal{A}_{4}|r| L,
$$

where

$$
\tau_{r}=\left(\mathcal{A}_{3}|r|^{3} L\right)^{1 /(\Delta-\nu)}
$$

and we have used the exponent relation [11] $\gamma=\Delta-\beta$ to introduce the gap exponent $\Delta$. Once again it is understood that the exponents take their classical values. In the region of validity of approximation (1.5), i.e. for $1 \gg \tau \gg \tau_{0}$, with $\xi\left(\tau_{0}\right) \sim L$ three different ranges of $\tau_{r}$ with qualitatively different behaviours of $\Gamma(\tau)$ can be distinguished [6]:

1. $\tau_{r} \ll \tau_{0}$

In this case the first term in square brackets in (2.7) dominates in the whole region of validity of this approximation since $\tau / \tau_{r} \gg 1$ and $\nu-\Delta$ is negative. Hence, in this region the adsorption should increase monotonically as $\tau \rightarrow 0$.

2. $\tau_{r} \gg 1$

This condition is equivalent to $|r|^{3} L \gg 1$. In this case $\tau / \tau_{r} \ll 1$ throughout the critical region and the second term in square brackets in (2.7) dominates. Hence, the adsorption is negative and desorption takes place despite the presence of adsorbing walls.

3. $\tau_{0} \ll \tau_{r} \ll 1$

For a given pore width $L$, the second term in square brackets in (2.7) dominates so long as $\tau<\tau_{r}$ and then $\Gamma(\tau)$ is negative. As $\tau$ increases, $\Gamma(\tau)$ reaches a maximum for $\tau \approx \tau_{r}$. Finally for $\tau \gg \tau_{r}$ the constant term in square brackets in (2.7) dominates over the second term, and for such temperatures the usual Fisher - de Gennes type of adsorption should occur.

We now consider values of parameters appropriate to the experiments of Ref. [1.2]. Assume that $L / \sigma \sim 10^{2}$, is of the size of the nominal pore diameter ( $\sigma$ is the molecular size) of the mesoporous glass used as the adsorbent. For $r=-0.001,-0.005$, corresponding to the two near-critical isochores along which $\Gamma(\tau)$ was measured, $\tau_{r} \sim 10^{-7}, 10^{-5}$. For this value of $L, \tau_{0} \sim 10^{-4}$ and $\tau_{r} \ll \tau_{0}$. Then according to the above discussion the adsorption $\Gamma$ should increase monotonically as $T$ is lowered towards $T_{c}$ following these two isochores. Even for densities that deviate more strongly from $\rho_{c}$, e.g. $r=-0.01, \tau_{r} \sim 10^{-4} \sim \tau_{0}$ and the condition (3.) for the occurence of depletion of $\Gamma$ might still not be satisfied. It is also very likely that for these values of $r$ and $L$ the adsorption is positive for $\tau \approx \tau_{0}$ and hence for $\tau<\tau_{0}$ it should saturate at a positive value.

It is important to contrast this constant $r$ (density) scenario with the constant $H$ lattice gas described in Ref. [6]. There depletion was observed for fields $H$ in the range $-10^{-7}$ to $-1.5 \times 10^{-4}$. These were sufficiently strong to drive $\Gamma$ negative for $\tau>\tau_{0}$, i.e. while $\xi$ was smaller than $L$. $\Gamma$ then saturated at a negative value for $\tau \leq \tau_{0}$. In the present case, even for values of $r$ as negative as -0.01 , the effective field might not be strong enough to drive $\Gamma$ negative before $\xi \sim L$ and then $\Gamma$ would saturate at a positive value.

Our analysis so far has been based on (2.4). Consider now equations of state which do not incorporate the symmetry (2.5) in the $\mu-T$ plane. For systems described by such equations of state the 'true' scaling fields are now $u_{H}$ and $u_{\tau}$ and by analogy with bulk [10] the 'true' OP $m(z)$ which satisfies the scaling relations (1.1) and (1.2) is not $\rho-\rho_{c}$ but rather the linear combination of the number and entropy densities $\left(\rho-\rho_{c}\right)-c_{2}\left(s-s_{c}\right)$. The entropy term in the OP does not change the leading asymptotic behaviour of the adsorption for the semi-infinite system; it gives rise to a "correction term" to Eq.(1.2) of order $\tau^{1-\alpha-\nu}$, where $\alpha$ is the specific heat critical exponent. In the scaling analysis of $\Gamma$ for the confined system, the scaling field $H$ should be now replaced by $u_{H}$. In order to see if this can change the 
behaviour of $\Gamma$ we first consider equations of state that are linear in $\tau$, as was the case in the vdW equation of state. For all such equations the 'mixed' scaling field $u_{H}$ reduces to $2 H$ defined by (1.3). This is due to the fact that the chemical potential on the critical isochore and the chemical potential at coexistence have the same limiting slope at $T_{c}$, i.e. $c_{1} / T_{c}=\lim _{T \rightarrow T_{c}^{-}}\left(d \mu^{\text {coex }} / d T\right)=\lim _{T \rightarrow T_{c}^{+}}(\partial \mu(\rho, T) / \partial T)_{\rho_{c}}$ so that

$$
u_{H}=\mu(\rho, T)-\mu_{c}-c_{1} \tau=\Delta \mu+\mu\left(\rho_{c}, T\right)-\mu_{c}-c_{1} \tau=\Delta \mu=2 H .
$$

Thus, for the classical equations of state which are linear in temperature the reduced symmetry of the fluid does not influence the temperature behaviour of the bulk field. $2 H=\Delta \mu$ differs from the leading order behaviour only by terms higher in $r$. For example, in the case of the vdW equation of state these are of order $r^{4}$ and $\tau r^{3}$.

If the equation of state is not linear in temperature then

$$
u_{H}=\Delta \mu+\mu\left(\rho_{c}, T\right)-\mu_{c}-c_{1} \tau=\Delta \mu+a_{2} \tau^{2}+O\left(\tau^{3}\right),
$$

where $a_{2}$ is a constant coefficient. Thus, the temperature dependence of the scaled field $u_{H}$, which now plays the role of the bulk field $H$, differs from that of $\Delta \mu$, but only by terms higher order in $\tau$.

We conclude that differences arising from the reduced symmetry of the fluid, i.e. 'mixed' scaling fields, are not important for the behaviour of the adsorption. The presence of higher order terms in $r$ and $\tau$ do not change the conclusions of our analysis performed using (2.4). Thus our predictions of no depletion of adsorption along nearcritical isochores should be valid for all classical equations of state.

Of course real fluids are non-classical. Our argument can be extended using the fact that near criticality real fluids should obey the scaled equation of state 12

$$
\Delta \mu=r|r|^{\delta-1} D_{0} h\left(\tau /|r|^{1 / \beta}\right)
$$

where $\delta$ is the critical exponent and $D_{0}$ is an amplitude for the power law on the critical isotherm, and $h(x)$ is a scaling function. Note that $\Delta \mu=\mu(\rho, T)-\mu^{\operatorname{coex}}(T)$ for $\tau<0$ and $\delta=1+\gamma / \beta$. Although there is no a priori theoretical expression for $h(x)$ the scaling function should satisfy several conditions following from requirements of thermodynamic stability and analyticity of the chemical potential. Thus, $h(x)$ should be analytic in its range of definition $-1<x<\infty$, equal to 0 at $x=-1$, the coexistence curve, and possess an (asymptotic) series expansion near $x=\infty$ (the critical isochore) of the form

$$
h(x)=\sum_{n=1}^{\infty} \eta_{n} x^{\beta(\delta+1-2 n)} .
$$

For small values of $x, h(x)$ should have an expansion of the form

$$
h(x)=1+\sum_{n=1}^{\infty} h_{n} x^{n} .
$$

The leading temperature dependence of $\Delta \mu$ on the near-critical isochores is given by the first term in expansion (2.12) and the leading $r$ dependence is given by the first term in expansion (2.13), i.e.

$$
\Delta \mu \sim \eta_{1} r \tau^{\gamma}+D_{0} r|r|^{\delta-1}
$$

where $D_{0}$ and $\eta_{1}$ are amplitudes. For classical exponents this expression is consistent with Eq.(2.4). Using this form for the bulk field $2 H \sim \Delta \mu$ we can repeat the analysis performed above. Eq. (2.7) remains valid but now $\tau_{r} \sim\left(|r|^{\delta} L\right)^{1 /(\Delta-\nu)}$. For real fluids $\delta \sim 4.78$ which means that the values of $\tau_{r}$ are even smaller for a given $r$ than in the classical case. This implies that the effective bulk field is very weak for the conditions of the experiment and thus depletion of the adsorption $\Gamma$ should not occur. Rather saturation of $\Gamma$ at positive values should be expected.

\section{RESULTS FROM DENSITY FUNCTIONAL THEORY}

In this section we report the results of density functional calculations for the adsorption $\Gamma$ of a near-critical, simple fluid confined in a slit-like pore. These results provide an explicit test of the heuristic ideas given above.

Specifically, we consider a fluid confined between two identical parallel adsorbing walls located at $z=0$ and $z=L$ and infinite in the $x$ and $y$ directions. The system is in contact with a bulk reservoir at fixed temperature $T$ and chemical potential $\mu$. The equilibrium profile is obtained by minimizing the grand potential functional [13] 


$$
\Omega[\rho]=\mathcal{F}[\rho]-\int d \mathbf{r}(\mu-V(\mathbf{r})) \rho(\mathbf{r}),
$$

where $V(\mathbf{r})$ is the total wall-fluid external potential

$$
V(\mathbf{r}) \equiv V(z)=U(z)+U(L-z)
$$

and $U(z)$ is the solid-fluid potential due to a single wall. The equilibrium density profile $\rho(\mathbf{r}) \equiv \rho(z)$ corresponds to the minimum of $\Omega[\rho]$. We choose the simplest form for $\Omega$ based on the square gradient approximation to the intrinsic free energy functional $\mathcal{F}[\rho]$ and model the wall-fluid contribution by a term $\Phi_{s}$ which depends only on the fluid density at contact i.e. on $\rho(0)=\rho(L)$. In this approximation the grand-potential excess per unit area is the following functional [14]:

$$
\gamma[\rho]=\frac{1}{2}\left(\int_{0}^{L} d z\left[\psi(\rho)+\frac{D}{2}\left(\frac{d \rho}{d z}\right)^{2}\right]+\Phi_{s}\right) .
$$

Here $\psi(\rho) \equiv \omega(\rho)+P$ is the excess grand-potential density, i.e. $\omega(\rho) \equiv f(\rho)-\mu \rho$ is the grand potential density, $P$ is the pressure and $f(\rho)$ is the Helmholtz free energy density of a homogenous fluid of density $\rho$. For $T<T_{c} \psi(\rho)$ has two minima corresponding to the two distinct bulk phases. At bulk coexistence both minima are equal to zero. The coefficient $D$ is related to the second moment of the direct correlation function [13] but for simplicity we choose it to be density independent. The wall-fluid term has the form

$$
\Phi_{s}=\frac{c}{2}\left(\rho^{2}(0)+\rho^{2}(L)\right)-\varepsilon_{w}(\rho(0)+\rho(L)) .
$$

The first term, with $c>0$, represents a reduction of attractive pair interactions between fluid particles at the surface arising from exclusion of the fluid by a wall. The second term with $\varepsilon_{w}>0$ measures the strength of the attractive wall potential. Symmetry of the wall-fluid potential dictates that $\rho(0)=\rho(L)$ and $d \rho / d z=0$ at $z=L / 2$.

It is well known that functionals of this type cannot incorporate short-ranged correlations and hence cannot acount for oscillations of the density profile which occur near the walls [8]. However, they should capture the main features of critical adsorption in large pores, as this phenomenon is dominated by the behaviour of the profile far from the walls. Indeed they were succesfully employed by Marini Bettolo Marconi 15] in a pioneering study of the effects of finite size on critical adsorption.

Minimization of (3.3) yields the following equation for the density profile $\rho(z)$ :

$$
D \frac{d^{2} \rho(z)}{d z^{2}}=\frac{d \psi}{d \rho(z)}
$$

with boundary condition at the wall $z=0$,

$$
D\left[\frac{d \rho(z)}{d z}\right]_{z=0}=c \rho(0)-\varepsilon_{w} .
$$

Eq.(3.5) has a first integral

$$
\frac{D}{2}\left[\frac{d \rho(z)}{d z}\right]^{2}=\psi(\rho)+F
$$

where $F$ is a constant of integration, independent of $z$, whose value depends on $T, L$ and $\mu$. The function $F(L)$, which vanishes as $L \rightarrow \infty$, can be identified with the solvation force between the walls [15, 16] i.e. $F(L)=-2(\partial \gamma / \partial L)_{T, \mu}$, where $\gamma$ is the equilibrium value of $\gamma[\rho]$. It may be determined from the equations

$$
L=(2 D)^{1 / 2} \operatorname{sgn}(\rho(0)-\rho(L / 2)) \int_{\rho(L / 2)}^{\rho(0)} \frac{d \rho}{[\psi(\rho)-\psi(\rho(L / 2))]^{1 / 2}}
$$

and

$$
F=-\psi(\rho(L / 2)
$$

both of which follow from Eq.(3.7) along with 


$$
\psi(\rho(0))-\psi(\rho(L / 2))=\frac{1}{2 D}\left(c \rho(0)-\varepsilon_{w}\right)^{2}
$$

which follows from the boundary condition (3.6). The key quantity of this study, the Gibbs adsorption $\Gamma_{G}$ (coverage) is defined as

$$
\Gamma_{G}=\int_{0}^{L}\left(\rho(z)-\rho_{b}\right) d z
$$

with $\rho_{b}$ the density of the bulk fluid at chemical potential $\mu$ and temperature $T . \Gamma_{G}$ satisfies the Gibbs adsorption equation

$$
\Gamma_{G}=-2(\partial \gamma(L) / \partial \mu)_{T},
$$

which gives, using Eq.(3.3), the following expression for $\Gamma_{G}$

$$
\Gamma_{G}=(2 D)^{1 / 2} \operatorname{sgn}(\rho(0)-\rho(L / 2)) \int_{\rho(L / 2)}^{\rho(0)} \frac{d \rho\left(\rho(z)-\rho_{b}\right)}{[\psi(\rho)-\psi(\rho(L / 2))]^{1 / 2}} .
$$

In order to test our predictions from Sec.2 we chose three different models for $\psi(\rho)$ and calculated the adsorption as a function of temperature on approaching $T_{c}$ from above along near-critical isochores, i.e. for fixed $\rho \leq \rho_{c}$.

(a) Landau model free energy.

In this case we expand the grand potential density $\omega(\rho)$ and the pressure $P=-\omega\left(\rho_{b}\right)$ about the critical density $\rho_{c}$. In terms of reduced variables $r=\left(\rho-\rho_{c}\right) / \rho_{c}$ and $r_{b}=\left(\rho_{b}-\rho_{c}\right) / \rho_{c}$ the dimensionless excess grand potential is

$$
\psi^{*}(r)=\frac{a^{*}}{2}\left(r^{2}-r_{b}^{2}\right)+\frac{b^{*}}{4}\left(r^{4}-r_{b}^{4}\right)-\left(r-r_{b}\right) \Delta \mu^{*},
$$

where $\psi^{*} \equiv \psi(\rho) / k_{B} T_{c} \rho_{c},\left(k_{B} T_{c} / \rho_{c}\right) a^{*} \equiv a=(\partial \mu / \partial \rho)_{T}$ at $\rho=\rho_{c},\left(k_{B} T_{c} / \rho_{c}^{3}\right) b^{*} \equiv b=\frac{1}{6}\left(\partial^{3} \mu / \partial \rho^{3}\right)_{T}$ at $\rho=\rho_{c}$ and $\Delta \mu^{*}=\left(\mu-\mu\left(\rho_{c}, T\right)\right) / k_{B} T_{c}[18]$.

Such a choice for $\psi(\rho)$ corresponds to the simplest mean-field or Landau description of a model fluid exhibiting particle-hole symmetry. For the special case $\Delta \mu=0$ (on the critical isochore $\rho_{b}=\rho_{c}$ ) the integrals (3.8) and (3.13) can be performed explicitly in terms of the incomplete Jacobi elliptic integral of the first kind [24]. For $\Delta \mu<0$, i.e. for $\rho_{b}<\rho_{c}$, the relation (3.8) between the order parameter at the midpoint $r_{m}$ and the wall separation $L$ can also be expressed in terms of the elliptic integral of the first kind (see Appendix).

For a given value of the separation $L$ between the walls and for fixed bulk density $\rho_{b}$, we determined the reduced densities $r_{m}$ and $r_{w}$ and $F(L)$ at various temperatures, corresponding to $\tau$ between 0.1 and 0 , using a graphical construction [16] along with Eq.(3.8). For a given $L$ there is only one solution in this range of temperatures. At each temperature we calculate numerically the integral (3.13) for $\Gamma_{G}$ using the Romberg method. As a check of the accuracy we calculated $\Gamma_{G}$ for $r_{b}=0$ using analytical expressions for the integrals (3.8) and (3.13)in terms of the incomplete Jacobi elliptic integral of the first kind.

We performed our calculations using parameters $a^{*}$ and $b^{*}$ obtained by fitting the critical temperature and the critical density of $\mathrm{SF}_{6}$, i.e. $\rho_{c}=3.05 \mathrm{~nm}^{-3}, T_{c}=318.7 \mathrm{~K}$ using a generalized vdW equation of state (see Ref. 15] for details). We find $a^{*}=2.764 \tau$ and $b^{*}=0.113$. The value of $D$ was taken from Ref. [15], giving $D^{*}=1.094$. The wall fields $c$ and $\varepsilon_{w}$ were treated as independent parameters which were varied in order to examine the influence of the strength of the wall potential on the behaviour of $\Gamma_{G}(\tau)$.

We studied systems with different wall separations $L$ ranging between 25 and $200 \mathrm{~nm}$. For each $L$ and fixed wall fields $c$ and $\varepsilon_{w}$ we calculated $\Gamma_{G}(\tau)$ along the critical isochore and for several near-critical isochores, i.e. for fixed $r_{b}$ between 0 and -0.1 .

The results for $L=100 \mathrm{~nm}, c^{*} \equiv c / D=0.5 \mathrm{~nm}^{-1}$ and $\varepsilon_{w}^{*} \equiv \varepsilon_{w} /\left(\rho_{c} D\right)=1 \mathrm{~nm}^{-1}$ and several values of $r_{b}$ are shown in Fig.1A. Similar behaviour of the adsorption is found for the other values of $L$ we considered. Note that the effective hard-sphere diameter resulting from fitting the $\mathrm{SF}_{6}$ data is $0.433 \mathrm{~nm}$ [15]. In order to allow for a more direct comparison with the analysis of Sec.2 and with the results from the lattice gas (Ising) model of Ref. [6] we plot in Fig. 1 the quantity

$$
\Gamma_{c} \equiv \int_{0}^{L} d z\left(\rho(z)-\rho_{c}\right)=\Gamma_{G}+\rho_{c} r_{b} L
$$


rather than Gibbs adsorption $\Gamma_{G}$. Our choice for the coefficients $c^{*}$ and $\varepsilon_{w}$ corresponds to walls that attract fluid rather strongly. On the critical isochore, i.e for $r_{b}=0$, the reduced contact density $r_{w} \approx 0.57$ at $T=T_{c}$ and does not change very much as the temperature is increased. Our results show no depletion; for all choices of the the bulk density the curves $\Gamma_{c}(\tau)$ increase monotonically with decreasing $\tau$. The adsorption first increases as $T$ approaches $T_{c}$ from above and then saturates sufficiently close to $T_{c}$. Except for $r_{b}=-0.1$, the value at saturation is positive. Moreover for the isochores which are the nearest to the critical one, i.e. for $0 \leq r_{b} \leq-0.01$, the values of $\Gamma_{c}(\tau)$ are very close for $\tau<10^{-4}$ and the adsorption saturates at $\tau \approx 10^{-5}$ corresponding to $\xi \sim L$, which agrees with the result found from the Ising model [6]. We have examined the behaviour of $\Gamma_{c}(\tau)$ for several choices of the coefficients $c^{*}$ and $\varepsilon_{w}^{*}$ but no qualitative differences in behaviour have been found. When the strength of the wall-fluid interaction $\varepsilon_{w}^{*}$ decreases, the degree of adsorption becomes smaller.

(b) Lennard-Jones fluid free energy.

As a second model we chose $f(\rho)$ to be the Helmholtz free energy density of the Lennard-Jones (LJ) fluid as given by the empirical, modified Benedict-Webb-Rubin (MBWR) equation of state [9]:

$$
f^{*}\left(\rho^{*}\right) \equiv \rho^{*} A^{*}\left(\rho^{*}\right)=\rho^{*}\left(\sum_{i=1}^{8} \frac{a_{i} \rho^{* i}}{i}+\sum_{i=1}^{6} b_{i} G_{i}\right)+\rho^{*} A_{i d}^{*}
$$

where $f^{*}\left(\rho^{*}\right) \equiv f(\rho) \sigma^{3} / \epsilon$ and $\rho^{*}=\rho \sigma^{3}$. $\epsilon$ is the LJ well depth and $\sigma$ is the LJ atomic diameter. $A_{i d}^{*}$ is the ideal gas term, $a_{i}$ and $b_{i}$ are nonlinear functions of temperature and $G_{i}$ are functions of the density $\rho^{*}$ (see Ref. [9]). The MBWR equation is a classical equation of state obtained by fitting to simulation data. It describes the near-critical region of a LJ fluid quite accurately. Since it is non-linear in temperature it does not possess the particle-hole symmetry of (2.5).

In order to calculate the adsorption in this model we proceed along the lines described above for the the Landau model but now we perform all the integrals numerically. We fit the parameters to the critical temperature and critical density of $\mathrm{SF}_{6}$ and the parameter $D$ is obtained from the relations $a=(\partial \mu / \partial \rho)_{T c}$ at $\rho=\rho_{c}$ and

$$
a / D=\xi_{0}^{-2} \tau^{2 \nu}
$$

with $\nu=1$, the classical value. $\xi_{0}$, the correlation-length amplitude, is set equal to the experimental value $0.2 \mathrm{~nm}$ for $\mathrm{SF}_{6}$. We find $D /\left(\sigma^{3} \epsilon\right)=0.364 \mathrm{~nm}^{2}$ and $\sigma=0.467 \mathrm{~nm}$.

Fig.1B shows the results for $\Gamma_{c}$ for the wall separation $L=50 \sigma$ and $r_{b}$ between 0 and -0.15 . The wall fields are chosen to be equal to $c^{*} \equiv c / D=0.5 \mathrm{~nm}^{-1}$ and $\varepsilon_{w}^{*} \equiv \varepsilon_{w} /\left(\rho_{c} D\right)=0.75 \mathrm{~nm}^{-1}$, which yields a contact density $r_{w} \approx 0.51$ at the critical point. The shapes of the curves are very similar to those for the Landau model. The absolute values of $\Gamma_{c}$ are smaller as $L$ is much smaller than the value used for the Landau model. Note that for this wall separation the density of the bulk reservoir must be further removed from $\rho_{c}$ in order to shift the whole adsorption curve below zero; for $r_{b}=-0.15, \Gamma_{c}$ still saturates at a positive value.

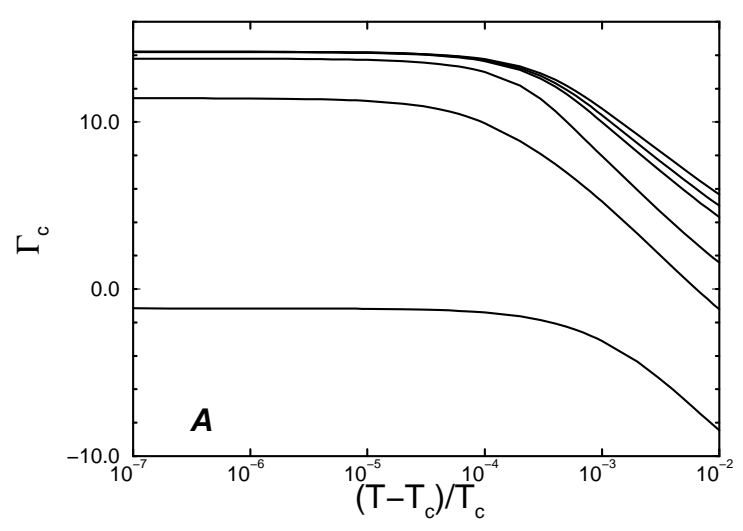



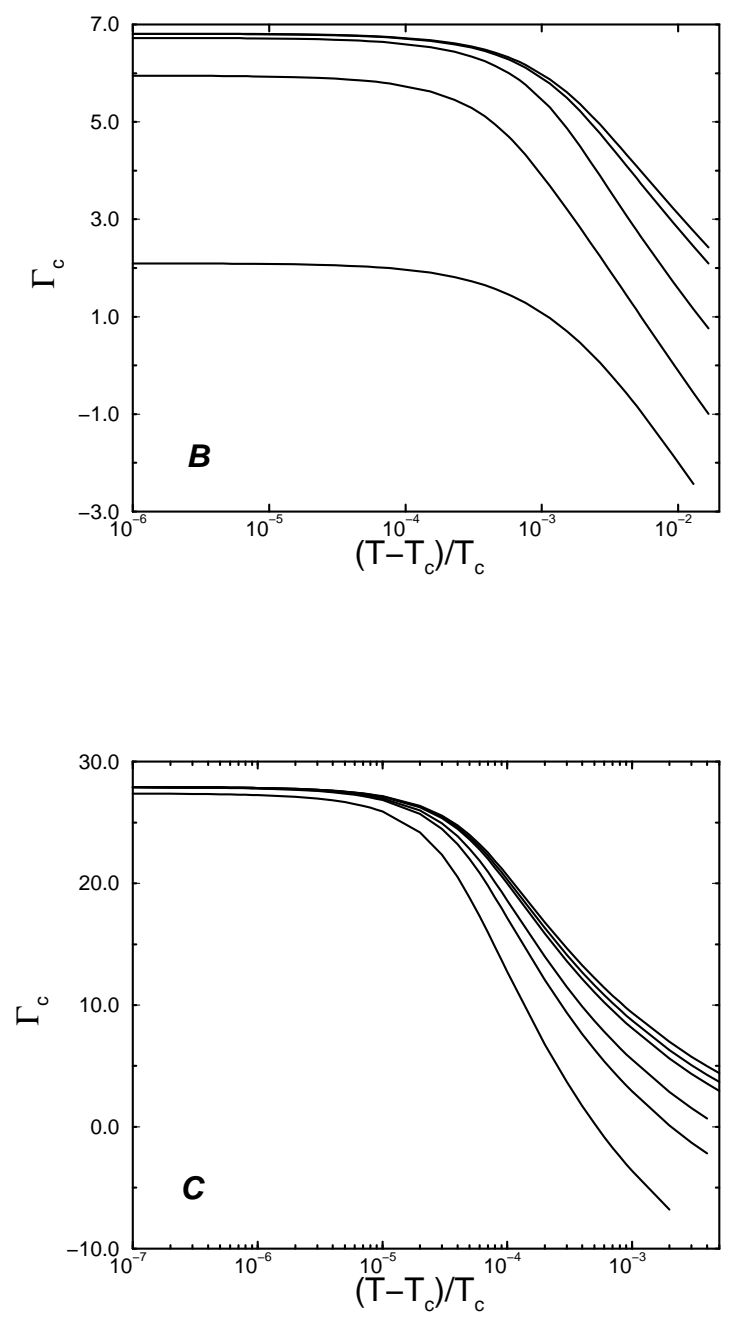

FIG. 1. Adsorption $\Gamma_{c}(\tau)$ (in units of $\mathrm{nm}^{-2}$ ) defined by Eq. (3.15) as a function of the deviation from the bulk critical temperature, calculated in three different models (A) Landau model for wall separation $L=100 \mathrm{~nm}$, surface field $\varepsilon_{w}^{*}=1$ $\mathrm{nm}^{-1}$ and surface coupling $c^{*}=0.5 \mathrm{~nm}^{-1}$. The curves correspond to different values of the reduced bulk reservoir density $r_{b}=\left(\rho-\rho_{c}\right) / \rho_{c}$. From the top to the bottom: $r_{b}=0,-0.005,-0.01,-0.03,-0.05,-0.1 ;$ (B) model with the free energy density of the LJ fluid for wall separation $L=50 \sigma$, where $\sigma$ is the LJ atomic diameter, surface field $\varepsilon_{w}^{*}=0.75 \mathrm{~nm}^{-1}$ and surface coupling $c^{*}=0.5 \mathrm{~nm}^{-1}$. The curves correspond to the following values of $r_{b}: r_{b}=0,-0.005,-0.01,-0.1,-0.15 ;(\mathrm{C})$ Fisk-Widom model for the same $L, \varepsilon_{w}^{*}, c^{*}$ and $r_{b}$ as in the Landau model.

(c) Fisk-Widom free energy.

In order to incorporate non-classical critical exponents we employ the simplest possible approach that goes beyond mean field, i.e. the Fisk - Widom functional [17]. This has the form of (3.3) with the grand potential density (3.14) replaced by

$$
\psi^{*}(r)=\frac{a^{*}}{2}\left(r^{2}-r_{b}^{2}\right)+\frac{b^{*}}{\delta+1}\left(r^{\delta+1}-r_{b}^{\delta+1}\right)-\left(r-r_{b}\right) \Delta \mu^{*},
$$

where $\psi^{*} \equiv \psi(\rho) / P_{c},\left(P_{c} / \rho_{c}^{2}\right) a^{*} \equiv a=(\partial \mu / \partial \rho)_{T}$ at $\rho=\rho_{c},\left(P_{c} / \rho_{c}^{\delta+1}\right) b^{*} \equiv b$ and $\Delta \mu^{*}=\left(\mu-\mu\left(\rho_{c}, T\right)\right) \rho_{c} / P_{c}$. The parameter $a$ now vanishes as the 'exact' inverse compressibility: $a \sim \tau^{\gamma}$ and the dimensionless quantities are defined in a slightly different way from those in the Landau model - see below (3.14). Following Ref. [15] we invoke rational approximants for the critical exponents, i.e. $\gamma=4 / 3, \nu=2 / 3, \beta=1 / 3$ and $\delta=5$. In this approximation the specific heat exponent $\alpha$ and the correlation function exponent $\eta$ are equal to zero and the coefficient $D$ can then be treated as a constant, whereas in reality $D$ diverges as $\tau^{-\eta \nu}$. We define $D^{*} \equiv D \rho_{c}^{2} / P_{c}$, which has dimension length ${ }^{2}$.

As for the Landau model, the integrals in the formulae for $L$ and $\Gamma_{G}$ can be expressed in terms of elliptic integrals of the first kind for the special case of the critical isochore $\Delta \mu=0$ (see Appendix). We use these formulae to calculate 
$\Gamma_{c}$ along the critical isochore. For $\Delta \mu \neq 0$ we evaluate the integral for $\Gamma$ numerically. The parameters $D^{*}, a^{*}$ and $b^{*}$ were obtained, following Ref. [15], from the Missoni-Levelt Sengers-Green nonclassical equation of state and from relation (3.17). These were fitted, as in the previous models, to the experimental values of $T_{c}, \rho_{c}$ and $\xi_{0}$ for $\mathrm{SF}_{6}$. The critical pressure $P_{c}=3.7605 \mathrm{MPa}$.

Fig.1C shows our results for $\Gamma_{c}$ calculated for the same wall separation $L=100 \mathrm{~nm}$, the same values of the wall fields $c^{*}=0.5 \mathrm{~nm}^{-1}$ and $\varepsilon_{w}^{*}=1 \mathrm{~nm}^{-1}$ and the same choices of the reduced bulk densities $r_{b}$ as the results obtained from the Landau model and shown in Fig.1A. Again, there is no depletion and the overall form of the results for the adsorption is similar to that for the Landau model and for the model with the LJ fluid free energy density. The adsorption is stronger since $\beta-\nu=-(1 / 3)$ for this model rather than 0 (logarithmic increase) for mean field. After saturation has set in, i.e. for $\tau \leq 2 \times 10^{-6}, \Gamma_{c}$ is almost independent of $r_{b}$. From the plot of the midpoint density $r_{m}$ as a function of temperature (Fig.2) we see that the temperature dependence of the adsorption along the isochores mimics the temperature dependence of $r_{m} . r_{m}$ takes on a value equal to $r_{b}$ for large $\tau$ and then increases as $\tau \rightarrow 0$. This behaviour is completely different from the case of the of the lattice-gas (Ising) model with constant negative bulk field in which the magnetisation becomes more negative as $T_{c}$ is approached from above.

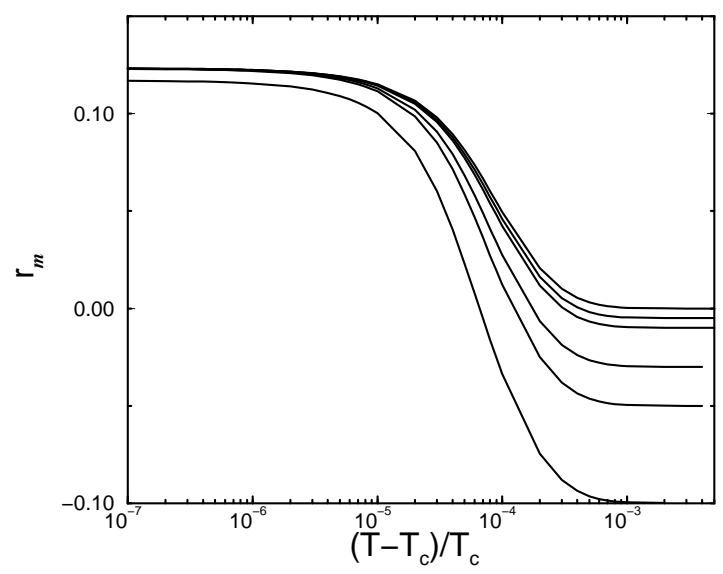

FIG. 2. The reduced midpoint density $r_{m}=\left(\rho(L / 2)-\rho_{c}\right) / \rho_{c}$ as a function of the deviation from the bulk critical temperature calculated in the Fisk-Widom model for the same parameters as in Fig. 1C.

\section{SIMULATION STUDIES OF A LENNARD-JONES FLUID}

\section{A. Computational details}

We have performed Monte Carlo simulations of a simple one component fluid, interacting via an interparticle potential of the Lennard-Jones (LJ) form:

$$
U_{L J}(r)=4 \epsilon\left[\left(\frac{\sigma}{r}\right)^{12}-\left(\frac{\sigma}{r}\right)^{6}\right]
$$

Here, as in Sec. III, $\epsilon$ measures the well depth of the potential, while $\sigma$ sets the length scale. As is customary in simulations of systems whose interparticle potential decays rapidly with particle separation, the LJ potential was truncated in order to reduce the computational effort. In accordance with many previous studies of the LJ system, the cutoff radius was chosen to be $r_{c}=2.5 \sigma$, and the potential was left unshifted. No corrections were applied to account for the effects of the truncation.

The simulations were performed within the grand canonical ensemble [19,20], permitting fluctuations in the total particle number $N$. Two distinct geometries were studied:

(A) A fully periodic cubic system of volume $V=L^{d}$.

(B) A slit-pore geometry in which the fluid is confined to a cuboidal simulation cell of dimensions $L_{x} \times L_{y} \times L$ (with $L_{x}=L_{y}$ ) having structureless hard walls in the planes $z=0$ and $z=L$, and periodic boundary conditions at the cell boundaries in the $x$ and $y$ directions parallel to the walls. 
Consider the behaviour of the configuration averaged local number density $\rho(\mathbf{r})$ in these systems. In geometry (A), translational invariance ensures that $\rho(\mathbf{r})$ is independent of the position vector $\mathbf{r}(x, y, z)$, and one has simply $\rho(\mathbf{r})=\rho$, i.e. the configuration averaged number density. In the absence of finite-size effects, $\rho$ is completely determined by the imposed values of the chemical potential $\mu$ and the temperature $T$. By contrast, in geometry (B) the presence of the walls at $z=0$ and $z=L$ break the translational symmetry in the $z$ direction giving rise to a one-dimensional density profile $\rho(\mathbf{r})=\rho(z)$ representing the configuration averaged local number density at a given $z$. The precise form of this profile depends not only on $\mu$ and $T$, but also on the details of the fluid-wall interaction. In this work, we assume that fluid particles interact with a single wall via a long ranged potential having one of either two forms:

$$
\begin{aligned}
U_{4-10}(z) & =4 \epsilon f_{4-10}\left[\frac{2}{5}\left(\frac{\sigma}{z}\right)^{10}-\left(\frac{\sigma}{z}\right)^{4}\right], \\
U_{3-9}(z) & =4 \epsilon f_{3-9}\left[\frac{2}{15}\left(\frac{\sigma}{z}\right)^{9}-\left(\frac{\sigma}{z}\right)^{3}\right],
\end{aligned}
$$

where $f$ is a parameter that tunes the strength of the fluid-wall interactions relative to those of the fluid interparticle interactions. The total wall-fluid potential is then given by (3.2). We note that $U_{4-10}(z)$ models a wall which is assumed to comprise a single plane of LJ particles, while $U_{3-9}$ models a wall that fills the half space [21]. Since both of these wall potentials decay considerably less rapidly with increasing separation than the LJ interparticle potential of eq 4.1, no potential truncation was applied.

It is instructive to compare the forms of the two types of fluid-wall potentials (eq. 4.2) both with one another, and with the Lennard-Jones (6-12) interparticle potential (eq. 4.1). This comparison is made in fig. 3 for the case $4 \epsilon=f=1$. The relative range of the two wall potentials is exposed by the inset which shows the result of first scaling the well depth of the 4-10 potential to equal that of the 3-9 potential, and then translating along the abscissa until both minima coincide. In this representation, one sees that the 3-9 potential is both longer ranged and exhibits a broader minimum than the 4-10 potential - a fact which will aid the interpretation of the simulation results presented below.

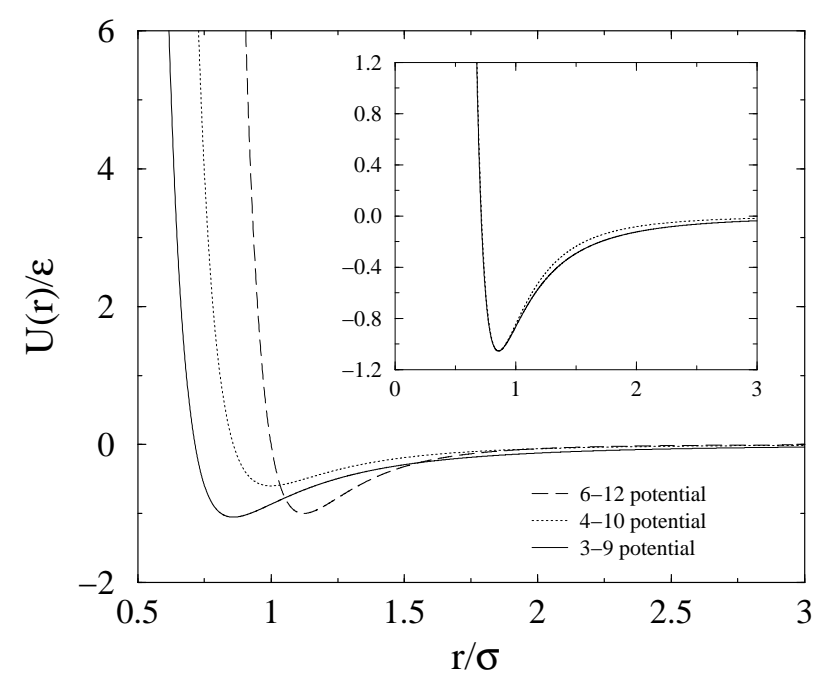

FIG. 3. Comparison of the potentials of eqs. 4.1 and 4.2 for the case $4 \epsilon=f=1$. The inset shows the result of scaling the well depth of the 4-10 potential to equal that of the 3-9 potential, and translating along the abscissa until the minima coincide.

\section{B. Determining the critical isochore}

As far as practicable, our simulation strategy has been to try to mimic the experimental adsorption studies of ref [1] in which $\Gamma(\tau)$ was measured along the bulk critical isochore as $T_{c}$ was approached from above. A prerequisite in this regard is an accurate knowledge of the locus of the bulk critical isochore. Within our grand canonical simulation framework, this is specified by the function $\mu\left(\rho_{c}, T\right)$. For the LJ fluid (with $\left.r_{c}=2.5 \sigma\right), \rho_{c}$ is known accurately from a previous finite-size scaling (FSS) study, as are estimates for $\mu_{c}$ and $T_{c}$ [22]. Hitherto, however, no accurate estimates for the critical isochore itself have been reported. Accordingly a new set of simulations were performed to determine 
$\mu\left(\rho_{c}, T\right)$ for a range of super-critical temperatures. These simulations were carried out using simulation geometry $(\mathrm{A})$ described above.

The procedure adopted for estimating $\mu\left(\rho_{c}, T\right)$ is detailed below and involves determining, for a given temperature $T$, that value of the chemical potential for which the measured density matches the known critical point value. Unfortunately this task is complicated by finite-size effects. Well away from the critical point the correlation length $\xi$ is small, and provided the linear dimension of the periodic simulation box $L \gg \xi$, the finite-size function $\mu_{L}\left(\rho_{c}, T\right)$ will provide a reliable estimate for the bulk isochore $\mu\left(\rho_{c}, T\right)$. As the critical point is approached, however, the correlation length grows until it is comparable to, or greater than the system size $L$. In this regime, one expects that estimates of $\mu_{L}\left(\rho_{c}, T\right)$ will deviate systematically from the limiting bulk form.

In principle, finite-size scaling (FSS) methods can be employed to obtain estimates for bulk quantities from simulations of finite size 23]. Unfortunately their application near the critical point is rather less straightforward than at the critical point. The difficulties stem from crossover effects associated with non-zero values of the two relevant scaling fields 10, $u_{H}$ and $u_{\tau}$, which control deviations of the number density and energy density from their critical point values-see Eqs. (2.1) and (2.2). Small but finite values of these fields result in a large but finite correlation length $\xi$ which, owing to computational restrictions on the range of accessible system sizes, renders the limit $L \gg \xi$ (in practical terms) unattainable. One is therefore forced to attempt to extrapolate to the thermodynamic limit using data from system sizes for which $L \lesssim \xi$. However, such an extrapolation is fraught with complications since it requires prior knowledge of the universal scaling functions (and associated non-universal amplitudes) controlling the crossover to the thermodynamic limit as both $\left|u_{\tau}\right|$ and $\left|u_{H}\right|$ are increased. To our knowledge, accurate forms for these scaling functions are not available.

In view of these difficulties, we have not attempted a full FSS analysis of the critical isochore. Instead we have simply determined $\mu_{L}\left(\rho_{c}, T\right)$ for the largest accessible system size and used this as our estimate for the bulk function $\mu\left(\rho_{c}, T\right)$. Notwithstanding the lack of a FSS analysis, there are formal grounds for believing that for the special case of the critical isochore, finite-size effects are smaller than on any other near critical isochore. To see this one must consider the effect on observables of finite values of the scaling field $\left|u_{H}\right|$. Specifically, we focus on the ordering operator conjugate to $u_{H}$, which is given by $\mathcal{M} \simeq\left(\rho-c_{2} s\right)$, representing some particular linear combination of the number and entropy densities 25$]$.

The dependence of $\delta \mathcal{M}=\mathcal{M}-\mathcal{M}_{c}$ on $\left|u_{H}\right|$ differs between the thermodynamic and FSS limits. For the former case one has $\delta \mathcal{M} \sim u_{H}^{1 / \delta}$, while for the latter, $\delta \mathcal{M}_{L} \sim u_{H} L^{\gamma / \nu}$. It follows that for the special case $u_{H}=0$, estimates of $\delta \mathcal{M}_{L}$ will agree with the bulk value of $\delta \mathcal{M}$ for all $L$, i.e. exhibit no finite-size dependence. For non-zero $\left|u_{H}\right|$, however, there will be a finite-size error $\delta \mathcal{M}_{L}-\delta \mathcal{M}$. Specifically, for a given $L$ one expects that as $\left|u_{H}\right|$ is increased from zero, $\delta \mathcal{M}_{L}-\delta \mathcal{M}$ initially increases like $\left|u_{H}\right|^{1 / \delta}$, but slows with increasing $\left|u_{H}\right|$, reaching a maximum at some $\left|u_{H}\right| \sim L^{-\beta \delta / \nu}$. Thereafter, further increase in $\left|u_{H}\right|$ lead to a decrease in the magnitude of $\delta \mathcal{M}_{L}-\delta \mathcal{M}$ until, for $\xi \ll L$, measurements of $\delta \mathcal{M}_{L}$ again agree with $\delta \mathcal{M}$. Thus as $\left|u_{H}\right|$ is increased from zero, the magnitude of the finite-size error $\delta \mathcal{M}_{L}-\delta \mathcal{M}$ associated with a given choice of $L$, first increases from zero, reaches a maximum and then falls back to zero.

As far as the number density itself is concerned, one finds 22 that on the line $u_{H}=0, \rho$ converges rapidly to its limiting value with increasing $L$ like $\rho_{L}-\rho \sim L^{-(1-\alpha) / \nu}$. For nonzero $\left|u_{H}\right|$, the dominant source of finite-size error is that described above. Thus for a given $L$, the finite-size error in the measured number density is minimised for state points on the line $u_{H}=0$, representing the analytical continuation of the coexistence curve to $T>T_{c}$. Given that the critical isochore and the line $u_{H}=0$ meet at the critical point and separate from one another only weakly as $T$ increases from $T_{c}$ (a feature that we have confirmed numerically), one can expect that the scaling field $u_{H}$ is generally very small on the critical isochore (see also Sec.2) and hence that the system size dependence of $\mu_{L}\left(\rho_{c}, L\right)$ is less than it would be on any sub- or super-critical isochore within the critical region.

To determine the finite-size critical isochore, the following procedure was employed. Taking a large, cubic, periodic simulation cell of linear size $L=17.5 \sigma$, runs were performed at the bulk critical point, for which [22] in reduced LJ units $T_{c}=1.1876(3), \mu_{c} / k_{B} T_{c}=-2.778(2), \rho_{c}=0.3197(4)$. At this density, the system contains, on average, $N=1715$ particles. Histogram reweighting [26] was then employed to estimate for $T=1.0028 T_{c}$, the $\mu$ value for which $\rho=\rho_{c}$. A second simulation was then performed at this new state point, followed by a further extrapolation of the results to obtain $\mu_{L}\left(\rho_{c}, T\right)$ for the still higher temperature of $T=1.015 T_{c}$. This procedure was then iterated a total of six times, giving isochoric data at temperatures $T=T_{c}, 1.0028 T_{c}, 1.015 T_{c}, 1.053 T_{c}, 1.087 T_{c}, 1.123 T_{c}$. Combining all six sets of simulation data via the multiple histogram reweighting scheme [26], it was then possible to map the entire isochore in the range $T_{c}<T<1.123 T_{c}$. Fig. 4 shows the results, together with the estimate of the critical isochore for a smaller system of linear size $L=10 \sigma$, determined using an identical procedure. The differences between these two estimates of $\mu_{L}\left(\rho_{c}, T\right)$ (inset of fig. 1 ) are less than one part in $10^{4}$, confirming the expectation that finite-size effects are small. 


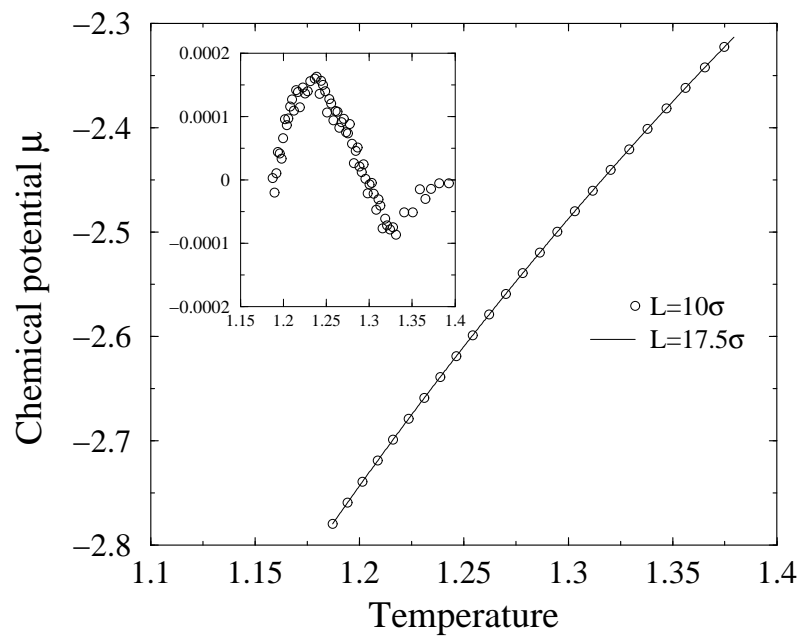

FIG. 4. The measured isochore $\mu_{L}\left(\rho_{c}, T\right)$ for the two periodic system sizes $L=17.5 \sigma$ and $L=10 \sigma$. The inset displays the difference between the two estimates. $\mu$ is the absolute chemical potential (in units of $k_{B} T$ ) subject to the convention of choosing the thermal wavelength $\lambda=1$ in the general definition (see Ref. 19)). Results are given in terms of LJ reduced units.

\section{Studies of the slit-pore geometry on the critical isochore}

Simulations of the LJ fluid confined to a mesoscopic slit pore (cf. section IV A ) have been carried out for state points along the critical isochore of fig. 4. Most of our studies are for a system of dimensions $L_{x}=L_{y}=15 \sigma, L=20 \sigma$, although some results have also been obtained for a system of size $L_{x}=65 \sigma, L=20 \sigma$ in order to gauge the magnitude of finite-size effects associated with the wall area $A=L_{x}^{2}$. We note that in the experiments of ref. [2] for $\mathrm{SF}_{6}$ in $\mathrm{CPG}$ glass, the pore diameter is about $31 \mathrm{~nm}$, corresponding to about 100 molecular diameters.

Simulation runs comprised $10^{5}$ Monte Carlo sweeps for equilibration followed by $5 \times 10^{6}$ sweeps for data collection. Measurements of the density profile were accumulated every 100 sweeps with each sweep involving $\left(L_{x} / r_{c}\right)^{3}$ attempted particle transfers and $\left(L_{x} / r_{c}\right)^{3}$ particle translations. Results have been obtained for both of the wall potentials given in equation (4.2), and for a range of values of the wall potential strength $f$.

\section{The critical point}

Density profiles $\rho(z)$ at the bulk critical parameters $\mu_{c}, T_{c}$ are shown in fig. 罒(a) for the 4-10 potential and fig. 国(b) for the 3-9 potential. In both cases the bulk critical density $\rho_{c} \sigma^{3}=0.3197$ is denoted by a horizontal dotted line.

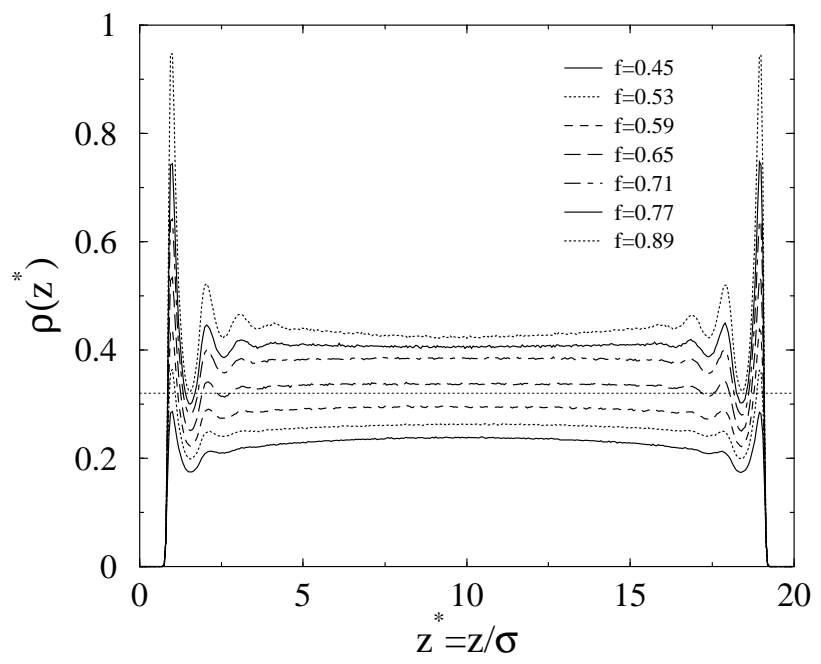




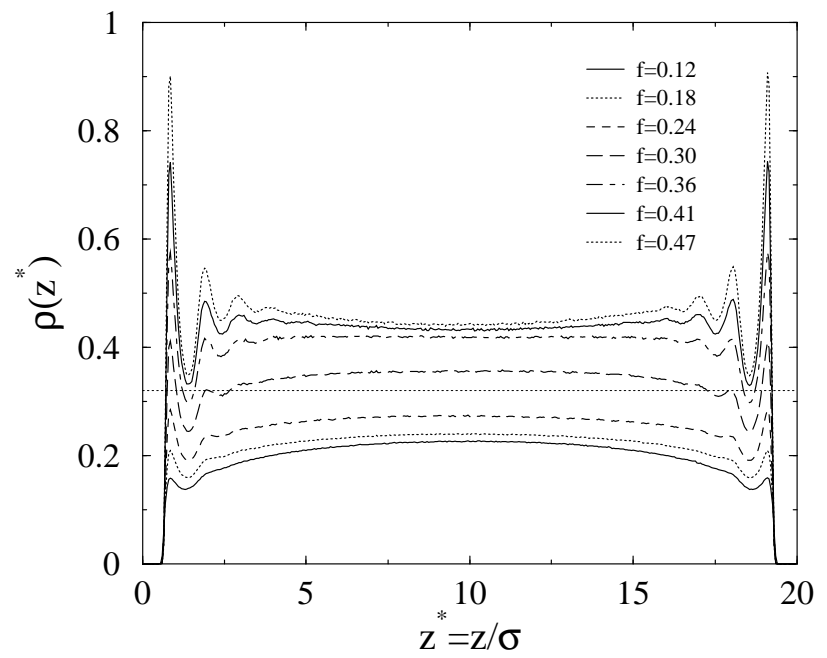

FIG. 5. (a) The measured density profiles $\rho(z)$ (in units of $\sigma^{3}$ ) for the 4-10 potential at the estimated values of the bulk critical parameters $\mu_{c}, T_{c}$. The system size is $L_{x}=15 \sigma, L=20 \sigma$. Results for a number of wall strengths $f$ are displayed (cf. eq (4.2)) (b) Same as (a) but for the 3-9 potential. In both cases the horizontal dotted line denotes the the critical density $\rho_{c}$.

It is instructive to compare and contrast the density profiles for both forms of wall potential. In all instances there is oscillatory structure close to the walls arising from excluded volume 'packing' effects. The number density at the wall (as measured e.g. by the height of the first peak) is principally controlled by the strength of the wall potential, i.e. by the value of $f$. Larger values of this parameter lead to a larger wall density and greater amplitude of oscillations in $\rho(z)$. Further away from the walls, the packing effects gradually die out and $\rho(z)$ varies smoothly with $z$. Within this smooth region, three main regimes of behaviour (common to both wall potentials) can be identified as $f$ is varied. We address them in turn.

For strongly attractive wall potentials $\left(f_{3-9} \geq 0.41, f_{4-10} \geq 0.77\right)$, the density at the wall greatly exceeds the critical density and the profile is convex downwards with respect to the critical density. Thus the local density $\rho(z)$ everywhere exceeds $\rho_{c}$ and decreases with increasing distance from the wall. At no point does it fall to $\rho_{c}$. This latter feature is in stark contrast to the simulation results of Schoen et al [4.5] who investigated a very similar model to that described here. For certain fluid-wall interaction strengths, they reported a density profile that greatly exceeds $\rho_{c}$ near the walls, but dips below $\rho_{c}$ in the slit middle. Recently, however, this depletion feature has been demonstrated to be an artifact [27], arising jointly from systematic errors in the simulation procedure used, and an incorrect designation of the critical point parameters $[28]$.

For weakly attractive fluid-wall interactions $\left(f_{3-9} \leq 0.24, f_{4-10} \leq 0.53\right)$ the density at the wall is less than the critical density and the profile is convex upwards with respect to the critical density. Thus the local density $\rho(z)$ is everywhere less than $\rho_{c}$ and increases with the distance from the wall. At no point does it attain the critical density. Tests performed at subcritical temperatures indicate that for $f$ values in this regime, the walls prefer the gas phase at coexistence. This is in contrast to the systems studied experimentally in ref. [1, 2], where the liquid wets the walls for $T<T_{c}$ and which, presumably therefore, correspond (in the language of the present model) to the large $f$ regime.

Turning now to intermediate wall strengths, the observed behaviour is somewhat subtle. Close to the wall, the packing-induced density oscillations span the critical density. Further away from the wall, the magnitude of the profile curvature is generally less than in the large or small $f$ limits. Interestingly, there exist $f$ values in this regime for which $\rho(z)$ exceeds $\rho_{c}$, but the profile is concave upwards with respect to the critical density. Thus $\rho(z)$ exceeds $\rho_{c}$ and increases with increasing distance from the wall. We shall return to discuss this unexpected finding in section $\nabla$.

Although both types of wall potential exhibit the same qualitative behaviour in the three regimes of $f$ described above, differences are present in the detail. This is particularly true for small values of $f$ as evidenced, for example, by a comparison of the profiles for $f_{3-9}=0.18$ and $f_{4-10}=0.53$. For these profiles the local densities in the slit middle are almost equal, but the wall density for the 4-10 potential is considerably greater than that for the 3-9 potential. This difference reflects the relative range of the two potentials (as discussed in section IV A) and in particular, the fact that to obtain a given magnitude of wall potential at the slit middle $(z=10)$, one requires $f_{4-10}=10 f_{3-9}$.

We round off this subsection with some remarks concerning finite-size effects associated with the finite wall area $A=L_{x}^{2}$. In a simulation, the infinite slit-pore limit $L_{x} / L \rightarrow \infty$ cannot be realised for all $L$ values of interest because of bounds on the computationally accessible system sizes. Well away from criticality, this should elicit no grave concern because periodic boundary conditions in directions parallel to the walls provide a good approximation to the thermodynamic limit. A critical system, on the other hand is always 'aware' of its boundary conditions, by virtue 
of its infinite correlation length. Changes in $L_{x}$ will therefore alter the effective range of correlations parallel to the walls, which might be expected to couple to those perpendicular to the walls in such as way as to affect the density profile $\rho(z)$. To investigate this possibility, we have performed simulations in which we increased $L_{x}$ from the value $L_{x}=15 \sigma$ considered hitherto, to $L_{x}=65 \sigma$. Owing to the high computational cost associated with such a large simulation cell, it was feasible to perform this comparison for only one type of wall potential, and we have chosen the 4-10 form. The results are shown in fig. 6. Comparison with those of fig. f(a) reveal that the increase in $L_{x}$ engenders only small changes in the form of the density profiles, the effect (such as there is) being greatest for intermediate wall strengths. On this basis it seems unlikely that the general scenario set out above would differ in the infinite slit limit. Nevertheless we feel that the role of wall area on confined critical systems is an issue that certainly merits a more systematic future investigation.

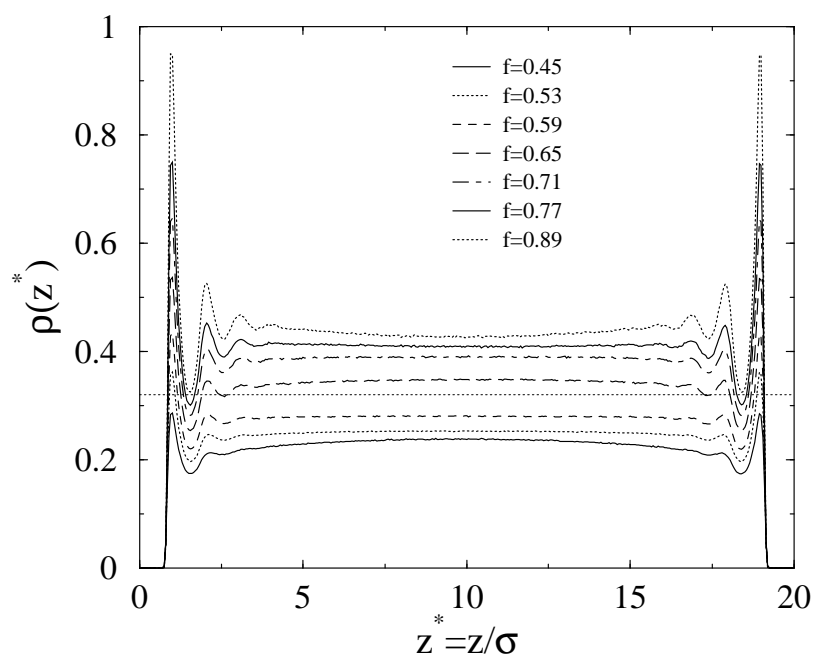

FIG. 6. As figure 5(a), but for a system having $L_{x}=65 \sigma, L=20 \sigma$.

\section{Super-critical temperatures}

In this subsection we present results for the temperature dependence of the density profile on the critical isochore. Both the 3-9 and the 4-10 wall potentials have been studied in this regard. However, since it transpires that the qualitative form of the results are similar in both cases, we describe only those results pertaining to the 4-10 potential.

Fig. 7 shows the forms of $\rho(z)$ at a selection of temperatures along the critical isochore of fig. 1, for the case $f_{4-10}=0.89$. This $f$ value represents a strongly attractive wall potential, as evidenced by the high wall density. From the figure, one observes that at the critical point the local density $\rho(z)$ is large compared to $\rho_{c}$ across the whole width of the slit. As the temperature is raised, however, the density in the slit middle decreases until, for $T \gtrsim 1.09 T_{c}$, it reaches the bulk value $\rho_{c}$. 


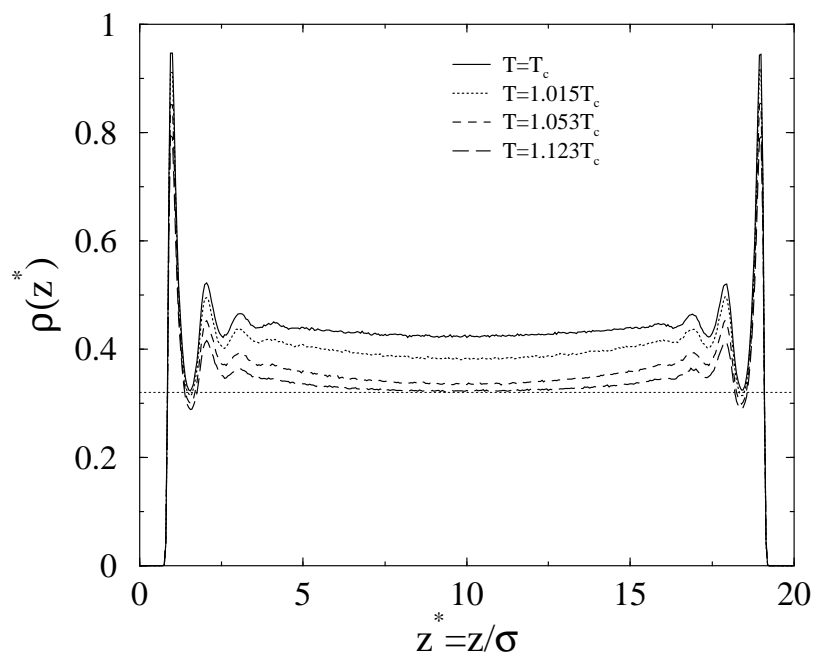

FIG. 7. The measured density profiles $\rho(z)$ (in units of $\sigma^{3}$ )for the $4-10$ potential with $f_{4-10}=0.89$ at a selection of temperatures along the critical isochore of figuref 4 . The critical density $\rho_{c}$ is denoted by a horizontal dotted line.

The explanation of this behaviour is straightforward. In the vicinity of the critical point, the correlation length exceeds the slit width $L$ and the density enhancement at the walls propagates across the whole slit, raising $\rho(z)$ with respect to $\rho_{c}$. In this regime one expects that for a sufficiently large slit width $L$, the density would decay to its critical value like $z^{-\beta / \nu}$, as is the case for critical adsorption at a single wall. Unfortunately our slit-pore is much too narrow for $\rho(z)$ to reach the bulk value in the available range of $z$. Neither do we observe pure power law scaling for the variation that is present. This is because there exists no substantial region of $z$ (away from the packing effects near the walls) which is not simultaneously influenced by the potentials of both walls.

As the temperature is increased, there is a concomitant decrease in the bulk correlation length $\xi \sim \tau^{-\nu}$ which at some point becomes less than the slit width $L$. For distances from the wall that exceed $\xi$, the decay of $\rho(z)-\rho_{c}$ is expected to crossover to an exponential form, $\exp (-z / \xi)$. We indeed observe a rapid relaxation of $\rho(z)$ to $\rho_{c}$ for high temperatures $\left(T \gtrsim 1.09 T_{c}\right)$, although for reasons similar to those described above, we have not been able to identify its character.

In order to quantify the temperature dependence of $\rho(z)$, and to make contact with the experimental studies of refs [1,2 and the theoretical results of section III, we have obtained the temperature dependence of the adsorption, $\Gamma_{c}$, defined by Eq. 3.15) The form of $\Gamma_{c}(\tau)$ is shown in fig. 8 for a representative selection of $f$ values. The observed behaviour falls naturally into 3 regimes of $f$ values, namely large, intermediate and small.

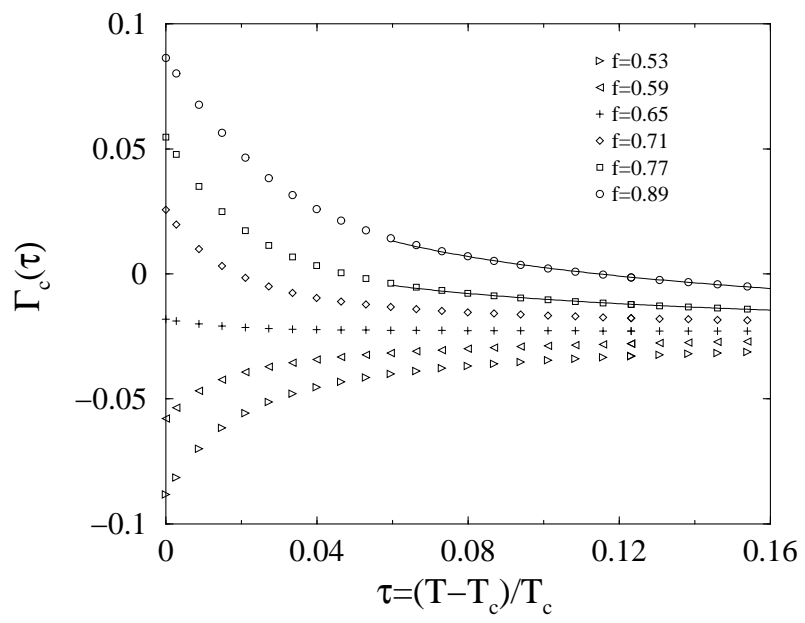


FIG. 8. The measured adsorption $\Gamma_{c}=\int_{0}^{L}\left(\rho(z)-\rho_{c}\right) d z$ ( in units of $\sigma^{2}$ ) for the 4-10 potential on the critical isochore, plotted as a function of the reduced temperature $\tau=\left(T-T_{c}\right) / T_{c}$. The slit width is $L=20 \sigma$. Data is shown for a selection of $f$ values, deriving from multi-histogram reweighting 26 of simulation data collected at six temperatures in the range $T_{c} \leq T \leq 1.123 T_{c}$. For the two largest $f$ values we also show fits to the data in the large $\tau$ regime, of the form $\Gamma(\tau)=a+b \tau^{\beta-\nu}$ with $\beta-\nu=-0.305$. Statistical errors do not exceed the symbol sizes.

For large $f\left(f_{4-10} \geq 0.77\right)$, fig. 8 shows that the adsorption increases monotonically as $\tau$ is reduced to zero from above. Values of $f$ in this range are believed to correspond to the situation studied experimentally in refs [1,2]. As was the case for DFT results of Sec. III, we find no evidence for the experimentally observed depletion phenomenon in which $\Gamma_{c}(\tau)$ first rises to a peak as $\tau$ decreases, and thereafter falls rapidly to negative values as the critical point is approached.

We have attempted to analyse the form of $\Gamma_{c}(\tau)$ for large $f$ at temperature well above criticality. Assuming there exists a regime for which $L \gg \xi \gg \sigma$, (with $\sigma$ the particle diameter), one expects that $\Gamma_{c}(\tau)$ will exhibit the universal scaling behaviour of critical adsorption at a single wall, i.e. $\Gamma_{c}(\tau) \sim \tau^{\beta-\nu}$. It is not clear, a-priori, that our rather narrow slit pore provides access to this regime. Nevertheless fig. 8 demonstrates that a fairly good fit to this form can be achieved in the high temperature regime.

As $f$ is reduced towards the intermediate regime, $\Gamma_{c}(\tau)$ becomes progressively flatter until, for $f_{4-10} \approx 0.65$, the adsorption appears to exhibit little or no temperature dependence. This 'neutral wall' scenario presumably arises from a (near) cancellation of two competing factors associated with the wall potential. On the one hand there is the 'missing neighbours' effect (represented by the parameter $c$ in equation (3.4)), whereby particles close to the wall have their potential energy raised relative to those in the bulk. On the other hand, there is the reduction in potential energy of particles arising from the attractive part of the wall potential (cf. the parameter $\epsilon_{w}$ in equation (3.4)). The cancellation of these two contributions effectively neutralises the influence of the wall on the fluid, and with it the temperature dependence of $\Gamma_{c}$. This is also clearly visible in the corresponding density profiles (fig. 9).

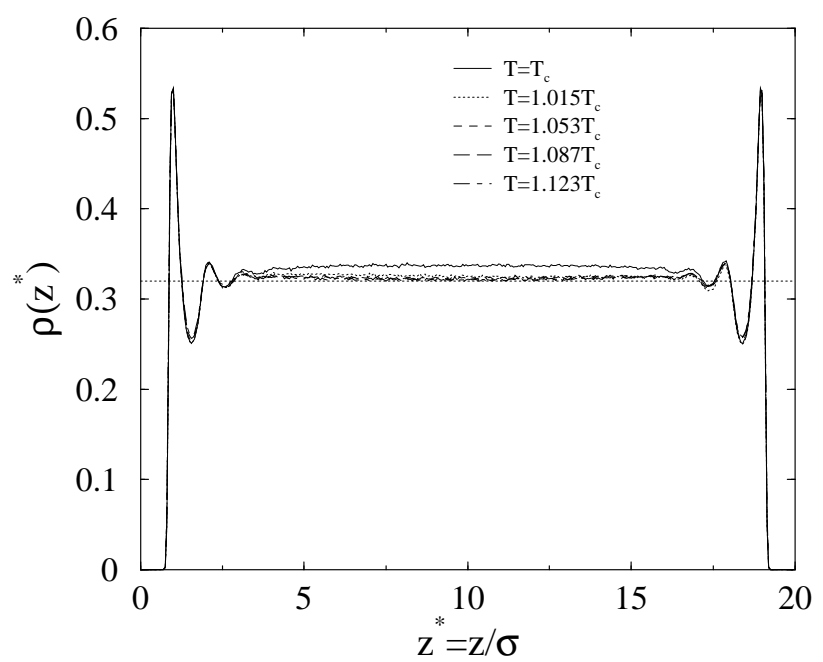

FIG. 9. The measured density profiles $\rho(z)$ ( in units of $\sigma^{3}$ ) for the $4-10$ potential with $f_{4-10}=0.65$ at a selection of temperatures along the critical isochore of figure 4 . The bulk density $\rho_{c}$ is denoted by a horizontal dotted line.

Turning finally to small $f$ values, we find that the adsorption is negative at the critical point, reflecting the fact (cf. fig. 5) that $\rho(z)<\rho_{c}$ for all $z$. As $\tau$ increases, however, $\Gamma_{c}(\tau)$ is observed to increase monotonically with increasing $\tau$, although it at no point becomes positive. This increase in $\Gamma_{c}$ is traceable to a progressive relaxation (away from the walls) of $\rho(z)$ towards the bulk value $\rho_{c}$. As in the case of large $f$ discussed above, this effect has its origin in the decrease of the correlation length.

\section{A sub-critical isochore}

In the experiments of refs [1.2], the adsorption was studied on sub-critical isochores having $0.995 \leq \rho / \rho_{c} \leq 0.999$. Unfortunately, in a simulation, it is not practicable to employ these same isochores because of the wholesale smearingout of the critical region by finite-size effects. Specifically, in the FSS regime, one finds [25] that $L^{\beta / \nu}\left(\rho-\rho_{c}\right) \simeq$ $\mathcal{F}\left(u_{H} L^{d-\beta / \nu}\right)$, where $\mathcal{F}$ is some scaling function. In practical terms, this means that applying a given non-zero 
bulk field to the fluid engenders a much smaller density change for a finite-size system than in the bulk limit. As a corollary, one finds that the typical scale of density fluctuations in a critical finite-sized system can be large. Thus for instance, for a cubic simulation cell of size $L=17.5 \sigma$, the critical point density fluctuations extend from $\rho \approx 0.15$ to $\rho \approx 0.5$. Clearly therefore a fractional reduction in the density of $0.5 \%$ would have little discernible effect, either on bulk finite-size or adsorption phenomena.

In view of this, we have chosen to study $\Gamma_{c}(\tau)$ on the isochore having $\rho=0.22 \sigma^{-3}=0.68 \rho_{c}$. Although this density is considerably smaller than $\rho_{c}$, it nevertheless lies within the range of critical point density fluctuations in our finitesized systems. One can therefore anticipate that if there is a critical depletion effect associated with negative values of the bulk field, then it should be visible on this isochore.

The locus of the $\rho \sigma^{3}=0.22$ isochore was determined in the temperature range $T_{c}<T<1.123 T_{c}$ using the same procedures as outlined in section IV B. However, an additional complication in the present case was a greatly increased finite-size dependence of $\mu_{L}(\rho, T)$. Comparison of $\mu_{L}(\rho, T)$ for $L=10 \sigma$ and $L=17.5 \sigma$ revealed finite-size effects an order of magnitude larger than those on the critical isochore (cf. inset of fig. 国). This feature was discussed in section IVB and is traceable to the differing magnitudes of the bulk field $u_{H}$ on each isochore. To ameliorate the problem, we determined $\mu(\rho, T)$ using a very large cubic system of linear size $L=40 \sigma$. Comparison of the results from $L=40 \sigma$ with those from a system of size $L=17.5 \sigma$ showed a discrepancy of less than two parts in $10^{4}$.

The form of $\Gamma_{c}(\tau)$ for the 4-10 potential on the $\rho \sigma^{3}=0.22$ isochore is presented in fig. 10. Data is shown for the same selection of $f$ values given in fig. 8. Comparing these two figures, it is clear that the application of the negative bulk field reduces the adsorption to a considerably greater degree at large $\tau$ than at small $\tau$. We attribute this to the large correlation length for small $\tau$, allowing the effect of the wall density (which is effectively pinned by the choice of $f$ ) to propagate across the slit, despite the action of the negative bulk field. The net effect is to increase the range of variation of $\Gamma_{c}(\tau)$ for large $f$ values but to reduce it for small $f$ values. The same qualitative behaviour is observed for the 3-9 wall potential. However, in no instance do we see any sign of a rapid critical depletion of the pore density as $\tau \rightarrow 0$.

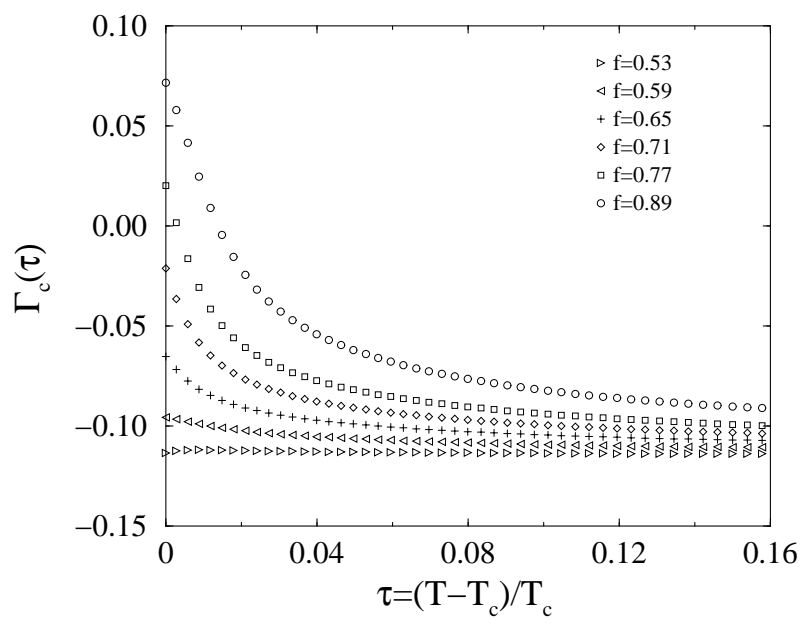

FIG. 10. The measured $\tau$ dependence of the adsorption $\Gamma_{c}=\int_{0}^{L}\left(\rho(z)-\rho_{c}\right) d z$ ( in units of $\sigma^{2}$ ) for the 4-10 wall potential on the $\rho \sigma^{3}=0.22$ isochore. Statistical errors do not exceed the symbol sizes.

\section{DISCUSSION}

The density functional (DFT) results and those of simulation have confirmed the heuristic arguments of Section II that there should be no critical depletion for a fluid in a single slit pore. In particular, for a fluid on its critical isochore, $\rho=\rho_{c}$, and for strongly attractive walls the adsorption $\Gamma_{c}$ increases monotonically as $T$ is reduced towards $T_{c}$. There is no evidence of the rapid decrease of $\Gamma_{c}$ near $T_{c}$ which is the experimentally observed signature of critical depletion. Although the simulation results, Fig.8, do not display the saturation of $\Gamma_{c}$ that is clear in DFT results of Fig. 2 we believe this reflects the different values of $L$ and the different temperature ranges used in the two treatments. In the DFT calculations, where $L$ is very large, saturation does not set in until $\tau \sim 10^{-5}$ and $\Gamma_{c}$ is still decreasing quite rapidly with $\tau$ for $\tau \geq 10^{-3}$. In the simulations, however, where $L=20 \sigma$ we would not expect saturation until $\tau \sim \tau_{0} \sim 3 \times 10^{-3}$ and this is difficult to discern on the scale of Fig.8. Such monotonic behaviour of $\Gamma_{c}$ is consistent with the lattice gas results for bulk field $H=0$ [6]. It differs from the Monte Carlo results of Schoen et.al. [4.5] but, as mentioned earlier, those simulations suffered from systematic errors [27,28]. The physical picture which emerges 
from both our present treatments is one in which the density profiles show increasing enhancement, with respect to $\rho_{c}$, in the central portion of the slit as the correlation length $\xi$ increases and the decay at each wall becomes slower. When $\tau \sim \tau_{0}$, i.e. $\xi \sim L$, there is maximum enhancement and maximum adsorption. Increasing the strength of the wall-fluid potential (the parameter $f$ ) simply raises the overall level of the profiles and leads to an increase in $\Gamma_{c}$ at a given value of $\tau$ - see Fig.8.

For weakly attractive walls (small values of $f$ ) the simulation results yield negative values of the adsorption on the critical isochore, with $\Gamma_{c}(\tau)$ monotonically increasing with increasing $\tau$. In this regime of $f$ the wall-liquid interface would be dry for $T<T_{c}$, i.e. the walls prefer gas to liquid. At supercritical temperatures the density near the wall is lower than $\rho_{c}$ and the profile increases towards the centre of the slit. The closer one is to $T_{c}$ the slower is the increase and the more negative is $\Gamma_{c}$. Similar behaviour of $\Gamma_{c}$ is found for very weakly attractive walls (small $\left.\epsilon_{w}\right)$ in our DFT calculations and was noticed earlier by Marini Bettolo Marconi [15]. By selecting an intermediate value of $f$ it is possible to construct a nearly 'neutral wall' for which $\Gamma(\tau)$ is small in magnitude and almost independent of $\tau$. In this case the density profiles are remarkably insensitive to $\tau$ until very close to $T_{c}$ where $\rho(z)$ has the striking form shown in Fig.9, i.e. it becomes concave upwards with respect to $\rho_{c}$. More careful analysis reveals that for slightly greater values of $f$ the profiles exhibit two symmetric maxima near the walls (but outside the regions of the oscillatory structure) for temperatures close to $T_{c}$. Similar, non-monotonic profiles were found recently for two-dimensional critical Ising films in the crossover regime between ordinary and normal transitions, i.e. for weak surface fields [29]. Currently we are investigating whether the non-monotonic form of the profiles found in the present simulations of a Lennard-Jones fluid arises from the same physical mechanism as in the Ising case.

We were motivated to investigate sub-critical isochores because i) the sorption experiments on porous glasses were performed for $r \equiv\left(\rho-\rho_{c}\right) / \rho_{c}=-0.001$ and -0.005 and ii) the lattice gas studies of Ref. [6, ,7] suggested that depletion should occur on a path at fixed bulk field $\Delta \mu=2 H<0$. Our present results for fluids show that no depletion occurs for the wide range of sub-critical isochores and pore-widths which we have investigated. The DFT results of Fig.2 imply that for the values of $r$ pertaining to the experiments the behaviour of $\Gamma_{c}(\tau)$ is changed very little from that on the critical isochore $r=0$. Increasing $|r|$ does alter $\Gamma_{c}(\tau)$ but this quantity remains monotonically increasing as $T \rightarrow T_{c}$. This is in keeping with the simulation results (with large $f$ ) of Fig. 10, although it should be noted that the simulations refer to $r=-0.31$ and to a much smaller value of $L$ than in the DFT calculations or in the experiments. In summary, our explicit calculations appear to confirm the expectations of Section II that, for strongly attractive walls and parameter values of practical interest, the effective bulk field in the fluid is insufficient to drive $\Gamma_{c}$ negative before $\xi \sim L$, i.e. we are in the regime $\tau_{r} \ll \tau_{0}$ where depletion does not occur. Alternatively one can say that for the relevant values of $r$, the experimental pore widths $L$ are not large enough for the density profile to relax from its high value near the walls to its bulk sub-critical value in the centre. Note that one cannot make $|r|$ too large or one leaves the experimental critical region. The circumstances of the fluid are different from those of the lattice gas, where for 'reasonable' choices of fixed bulk field $H$ one has [6] $\tau_{0} \ll \tau_{H}$, the parameter equivalent to $\tau_{r}$.

We conclude that the adsorption calculated along critical and sub-critical isochores for a single slit pore does not exhibit the depletion observed in the experiments. What then can be the explanation of the experimental data? One might speculate that for some reason the density of the reference cell, which fixes the experimental isochore, was not constant but it is still difficult to see why this might mimic the fixed $H$ scenario. What is more likely is that the observed depletion arises from the fact that the porous adsorbent is a complex solid material consisting of interconnected pores of various shapes and sizes whose morphology is poorly understood. Modelling such a material in terms of ideal (non-connected) slit pores is, of course, a gross over simplification. Recent theoretical and simulation studies [30] show that the phase behaviour of fluids confined in such disordered media can be very different from that which occurs in a single pore but we are not aware of investigations of the adsorption along near-critical isochores. It should be possible to employ the model porous glasses produced in the quench simulations of Gelb and Gubbins [31], which mimic the spinodal decomposition process used to make real CPG glasses, in such an investigation. Were the adsorption to be vastly different from that found for the 'average' single pore this would be a striking demonstration of the importance of pore disorder and connectivity for critical phenomena. Note that further evidence that the single pore model is inappropriate to describe the experiments comes from the observation that critical depletion occurs for the colloidal graphite substrate Vulcan $3 \mathcal{G}$ for $\rho / \rho_{c}=1.01$ and 1.04 i.e. for super-critical isochores [1,2]. Attempts to explain this observation within the single pore model require many further assumptions [6].

\section{ACKNOWLEDGMENTS}

A.M. acknowledges beneficial discussions with Alina Ciach. She also thanks the Royal Society/NATO for financial support. NBW acknowledges the financial support of the Royal Society (grant no. 19076), the EPSRC (grant no. GR/L91412) and the Royal Society of Edinburgh. 
${ }^{1}$ permanent address, Institute of Physical Chemistry, Polish Academy of Sciences, Kasprzaka 44/52, PL-01-224 Warsaw, Poland

[1] M. Thommes, G.H. Findenegg, H Lewandowski, Ber. Bunsenges. Phys. Chem. 98, 477 (1994).

[2] M. Thommes, G.H. Findenegg and M. Schoen, Langmuir 11, 2137 (1995).

[3] M.E.Fisher and P.G. de Gennes, C.R.Acad.Sci.Ser.B,287, 207 (1978).

[4] M. Schoen and M. Thommes, Phys. Rev. E 52, 6375 (1995).

[5] M. Schoen, M. Thommes, G.H. Findenegg, J. Chem. Phys 107, 3262 (1997).

[6] A.Maciołek, A.Ciach and R. Evans, J.Chem.Phys., 108, 9765 (1998).

[7] A. Drzewiński, A.Ciach and A.Maciołek, Europ.Phys.Journ. B, 5, 825 (1998).

[8] R.Evans, J.Phys.:Condens.Matter., 2, 8989 (1990) and references therein.

[9] J.K. Johnson, K.A. Zollweg and K.E. Gubbins, Mol. Phys., 78, 591 (1993).

[10] J.J. Rehr and N.D. Mermin; Phys. Rev. A. 8, 472 (1973).

[11] K.Binder, in Phase Transition and Critical Phenomena edited by C.Domb and J.Lebowitz ( Academic, London 1983),Vol 8 p.2.

[12] B. Widom, J.Chem.Phys., 43, 3898 (1965).

[13] R. Evans, Adv. Phys. 28, 143 (1979).

[14] J.W.Cahn, J.Chem.Phys., 66, 3367 (1977).

[15] U. Marini Bettolo Marconi, Phys.Rev. A, 38, 6267 (1988).

[16] R. Evans, U. Marini Bettolo Marconi and P.Tarazona, J.Chem.Phys., 84, 2376 (1985).

[17] S.Fisk, and B.Widom, J.Chem.Phys., 50, 3219 (1969).

[18] Note that this definition of the dimensionless $\Delta \mu^{*}$ differs slightly from that used in Sec.II for the vdW fluid.

[19] M.P. Allen and D.J. Tildesley Computer Simulation of Liquids, Oxford University Press (1987).

[20] D. Frenkel and B. Smit, Understanding Molecular Simulation, Academic Press, Boston (1996).

[21] J. Israelachvili Intermolecular and Surface Forces, Academic Press, London (1991).

[22] N.B. Wilding, Phys. Rev. E 52, 602 (1995).

[23] For a review see eg. V. Privman (ed.) Finite size scaling and numerical simulation of statistical systems (World Scientific, Singapore) (1990).

[24] Handbook of Mathematical Functions, edited by M. Abramowitz and I. A. Stegun (Dover, New York, 1964).

[25] N.B. Wilding and A.D. Bruce, J. Phys. Condens. Matter 4, 3087 (1992).

[26] A.M. Ferrenberg and R.H. Swendsen, Phys. Rev. Lett. 61, 2635 (1988); ibid 63, 1195 (1989).

[27] N.B. Wilding and M. Schoen; Phys. Rev.E, in press.

[28] In their study of the full LJ fluid in a slit pore, Schoen et al 国, fut quote the critical point parameters in LJ reduced units as being $T_{c}=1.36, \rho_{c}=0.365$. These values differ by up to several percent from those $\left(T_{c}=1.312(7), \rho_{c}=0.316(1)\right)$ recently determined by J.J. Potoff and A.Z. Panagiotopoulos; J. Chem. Phys. 109, 10914 (1998), in a FSS analysis of the same potential. We believe that the considerable overestimates of $T_{c}$ and $\rho_{c}$ in 4.5 accounts for their failure to see the slow critical point decay of $\rho(z)$ evident in fig. 5 for large values of $f$.

[29] A. Maciołek, A. Drzewiński and A. Ciach, Phys. Rev. E, (to appear)

[30] See the reviews by M.L.Rosinberg in 'New Approaches to Problems in Liquid State Theory' eds. C.Caccamo et.al. Nato Sci. Series C, Vol. 529 (Kluwer, Dordrecht, 1999) p.245 and L.D. Gelb, K.E. Gubbins, R. Radhakrishnan and M. SliwinskaBartkowiak, Rept. Prog. in Phys. (to appear).

[31] L.D. Gelb and K.E. Gubbins, Langmuir, 14,2097 (1998).

\section{APPENDIX: A}

For the Landau model the integrals $\sqrt{3.8}$ ) and $(3.13)$ can be performed explicitly in the special case of $\Delta \mu=0$ (on the critical isochore $\rho_{b}=\rho_{c}$ ) (see Ref. [15]):

$$
L=2 \rho_{c}^{-1 / 3}\left(\frac{D^{*}}{b^{*}}\right)^{1 / 2}\left(r_{m}^{2}+a^{*} / b^{*}\right)^{-1 / 2} F(\phi \mid m),
$$

where $r_{m}=\left(\rho(L / 2)-\rho_{c}\right) / \rho_{c}$ is the reduced density at the midpoint of the slit, and $D^{*} \equiv\left(D \rho_{c}^{5 / 3}\right) / k_{B} T_{c} . F(\phi \mid m)$ is the incomplete Jacobi elliptic integral of the first kind [24] with arguments $\cos \phi=r_{m} / r_{w}$, where $r_{w}=\left(\rho(0)-\rho_{c}\right) / \rho_{c}$ 
is the reduced density at the wall, and $m=\left(r_{m}^{2}+2 a^{*} / b^{*}\right) /\left(2 r_{m}^{2}+2 a^{*} / b^{*}\right)$. On the critical isochore $\rho_{b}=\rho_{c}$ the adsorption can also be evaluated [15]

$$
\Gamma_{G}=\rho_{c}^{2 / 3}\left(\frac{2 D^{*}}{b^{*}}\right)^{1 / 2} \ln \left|\frac{r_{w}^{2}+\frac{a^{*}}{b^{*}}+\left[r_{w}^{4}-r_{m}^{4}+\frac{2 a^{*}}{b^{*}}\left(r_{w}^{2}-r_{m}^{2}\right)\right]^{1 / 2}}{r_{m}^{2}+\frac{a^{*}}{b^{*}}}\right|-\rho_{c} r_{b} L .
$$

For $\Delta \mu<0$, i.e. for $\rho_{b}<\rho_{c}$, the relation (3.8) between the order parameter at the midpoint $r_{m}$ and the wall separation $L$ can also be expressed in terms of the elliptic integral of the first kind. For $T>T_{c}, a>0$ one can also show

$$
L=2 \rho_{c}^{-1 / 3}\left(\frac{2 D^{*}}{b^{*}}\right)^{1 / 2}(p q)^{-1 / 2} F(\phi \mid m)
$$

where the arguments of $F(\phi \mid m)$ are given by $\tan \phi / 2=\left(\frac{q\left(r_{w}-r_{m}\right)}{p\left(r_{w}-r_{1}\right)}\right)^{1 / 2}$ and $m=(1 / 4) \frac{(p+q)^{2}+\left(r_{m}-r_{1}\right)^{2}}{p q} \cdot r_{1}$ is the single real root of the cubic equation

$$
r^{3}+r^{2} r_{m}+r\left(r_{m}^{2}+2 a^{*} / b^{*}\right)+r_{m}^{3}+\left(2 a^{*} / b^{*}\right) r_{m}-\left(4 / b^{*}\right) \Delta \mu^{*}=0
$$

and $p^{2}=\left(u-r_{m}\right)^{2}+n^{2}, q^{2}=\left(u-r_{1}\right)^{2}+n^{2}$ where $u=-(1 / 2)\left(r_{1}+r_{m}\right)$ and $n^{2}=r_{m}^{2}-u^{2}-2 u r_{1}+2 a^{*} / b^{*}$.

Also for the case of Fisk-Widom free energy, the integrals in the formulae for $L$ and $\Gamma_{G}$ can be expressed in terms of elliptic integrals of the first kind for the special case of the critical isochore $\Delta \mu=0$ :

$$
L=\left(\frac{3 D^{*}}{b^{*} y}\right)^{1 / 2} \frac{1}{\lambda} F(\phi \backslash(\pi / 2-\alpha))
$$

where $y=r_{m}^{2}\left(\left(3 a^{*} / b^{*}\right)+r_{m}^{4}\right)$ and

$$
\begin{gathered}
\lambda^{2}=\left(\frac{-6\left(a^{*} / b^{*}\right)}{y r_{m}^{2}}+3 r_{m}^{-4}\right)^{1 / 2}, \\
\cos \phi=\left(\lambda^{2}-\frac{1}{r_{m}^{2}}+\frac{1}{r_{w}^{2}}\right) /\left(\lambda^{2}+\frac{1}{r_{m}^{2}}-\frac{1}{r_{w}^{2}}\right) \\
\sin ^{2} \alpha=1 / 2-(3 / 4) \frac{r_{m}^{-2}-a^{*} /\left(b^{*} y\right)}{\lambda^{2}}
\end{gathered}
$$

and

$$
\Gamma_{G}=\left(\frac{3 D^{*}}{b^{*}}\right)^{1 / 2} \rho_{c} \frac{1}{\lambda} F(\phi \backslash \alpha)-\rho_{c} r_{b} L
$$

where

$$
\begin{gathered}
\lambda^{2}=\left(3\left(a^{*} / b^{*}\right)+3 r_{m}^{4}\right)^{1 / 2}, \\
\cos \phi=\left(\lambda^{2}-r_{w}^{2}+r_{m}^{2}\right) /\left(\lambda^{2}+r_{m}^{2}-r_{w}^{2}\right) \\
\sin ^{2} \alpha=1 / 2-\frac{3}{4}\left(r_{m}^{2} / \lambda^{2}\right)
\end{gathered}
$$

Equivalent forms were derived in Ref. [15] where they were used to study the behaviour of the adsorption in the crossover regime from the noncritical to the scaling region as a function of wall separation and surface fields. 